\title{
NONRELATIVISTIC PARALLEL SHOCKS IN UNMAGNETIZED AND WEAKLY MAGNETIZED PLASMAS
}

\author{
Jacek Niemiec ${ }^{1}$, Martin Pohl ${ }^{2,3}$, Antoine Bret ${ }^{4,5,6}$, And Volkmar Wieland ${ }^{2,3}$ \\ ${ }^{1}$ Instytut Fizyki Jạdrowej PAN, ul. Radzikowskiego 152, 31-342 Kraków, Poland; jacek.niemiec@ifj.edu.pl \\ ${ }^{2}$ DESY, D-15738 Zeuthen, Germany \\ ${ }^{3}$ Institute of Physics and Astronomy, University of Potsdam, D-14476 Potsdam, Germany \\ ${ }^{4}$ Harvard-Smithsonian Center for Astrophysics, 60 Garden Street, MS-51, Cambridge, MA 02138, USA \\ ${ }^{5}$ ETSI Industriales, Universidad de Castilla-La Mancha, E-13071 Ciudad Real, Spain \\ ${ }^{6}$ Instituto de Investigaciones Energéticas y Aplicaciones Industriales, Campus Universitario de Ciudad Real, E-13071 Ciudad Real, Spain \\ Received 2012 July 13; accepted 2012 September 12; published 2012 October 17
}

\begin{abstract}
We present results of 2D3V particle-in-cell simulations of nonrelativistic plasma collisions with absent or parallel large-scale magnetic field for parameters applicable to the conditions at young supernova remnants. We study the collision of plasma slabs of different density, leading to two different shocks and a contact discontinuity. Electron dynamics play an important role in the development of the system. While nonrelativistic shocks in both unmagnetized and magnetized plasmas can be mediated by Weibel-type instabilities, the efficiency of shockformation processes is higher when a large-scale magnetic field is present. The electron distributions downstream of the forward and reverse shocks are generally isotropic, whereas that is not always the case for the ions. We do not see any significant evidence of pre-acceleration, neither in the electron population nor in the ion distribution.
\end{abstract}

Key words: acceleration of particles - instabilities - ISM: supernova remnants - methods: numerical - plasmas shock waves

Online-only material: color figures

\section{INTRODUCTION}

Collisionless shocks in space environments are generally considered to be sites of efficient particle acceleration. One of the main unsolved problems is how efficiently individual particles can be fed into a Fermi-type acceleration process. There, the particles have a mean free path, $\lambda_{\mathrm{mfp}}$, large enough that they see the shock as a sharp discontinuity and assume a power-law spectrum (Blandford \& Eichler 1987). By definition, collisionless shocks have a thickness of a few $\lambda_{\mathrm{mfp}}$ of the thermal ions. In particular for electrons, pre-acceleration appears to be required for injection into Fermi-type shock acceleration.

The shock transition region harbors small-scale electric and magnetic turbulence that dissipates the flow energy and may provide pre-acceleration (Bret 2009). Studies of nonrelativistic shocks indicate that the structure and dissipation mechanisms at these shocks vary considerably depending on the upstream plasma parameters and the angle between the magnetic field and the shock normal, $\theta_{\mathrm{Bn}}$. Quasi-perpendicular shocks, $\theta_{\mathrm{Bn}} \geqslant 45^{\circ}$, have been studied for many years (e.g., Papadopoulos 1988; Cargill \& Papadopoulos 1988; Lembège et al. 2004), and the basic structure of such shocks with Alfvén Mach number $M_{\mathrm{A}} \leqslant 10$ is well understood (e.g., Burgess \& Scholer 2007). However, the physics of quasi-perpendicular shocks in the limit of high Alfvén Mach number, as applicable to supernova remnants (SNRs), is still under debate, in particular the electroninjection problem (Umeda et al. 2009; Lembège et al. 2009; Amano \& Hoshino 2010; Kato \& Takabe 2010; Riquelme \& Spitkovsky 2011; Matsumoto et al. 2012).

In contrast, quasi-parallel shocks, $\theta_{\mathrm{Bn}} \leqslant 45^{\circ}$, have been harder to understand. Observations of magnetic-field-aligned ion beams and large-amplitude low-frequency magnetic fluctuations upstream of space-plasma shocks have incited interest in electromagnetic ion-beam instabilities (Gary 1984) as sources of the necessary dissipation at the shock, particle injection, and subsequent scattering into a Fermi-type acceleration mechanism (e.g., Quest 1988). Studies of the shock transition revealed that the shock ramp, where the principal density jump occurs, is not steady, but instead cyclically re-forms such that the shock on average maintains its correct speed (Winske et al. 1990; Krauss-Varban \& Omidi 1991). These effects have often been studied with one-dimensional hybrid simulations (Sugiyama 2011) that did not resolve electron and short-scale ion spatiotemporal scales. Short particle-in-cell (PIC) simulations of mildly relativistic quasi-parallel shocks were performed by Dieckmann et al. (2010) and Murphy et al. (2010). In quasiparallel conditions, Whistler and Alfvén waves are excited and in their nonlinear evolution grow into short large-amplitude magnetic-field structures, which are supposed to be efficient electron accelerators (Classen \& Mann 1997; Stasiewicz \& Ekeberg 2008; McClements \& Fletcher 2009).

Kato \& Takabe (2008) recently reported the formation of nonrelativistic shocks in unmagnetized electron-ion plasmas via a Weibel-type short-wave instability (Weibel 1959) for relative flow speeds between $0.2 c$ and $0.75 c$ (where $c$ is the speed of light). The relativistic two-stream Weibel (filamentation) instability (Medvedev \& Loeb 1999) is known to mediate relativistic-shock formation in collisionless unmagnetized or weakly magnetized electron-positron (e.g., Kazimura et al. 1998b; Silva et al. 2003; Nishikawa et al. 2003; Chang et al. 2008) and electron-ion (e.g., Kazimura et al. 1998a; Frederiksen et al. 2004; Spitkovsky 2008) plasmas. It leads to the development of current filaments and accompanying toroidal magnetic fields. The results by Kato \& Takabe (2008) (see also Stroman et al. 2012) demonstrate that a pre-existing magnetic field is not necessary for the existence of shock-mediating instabilities in nonrelativistic flows. They also show that electron dynamics are relevant for shock formation in unmagnetized plasma, which calls for a fully kinetic (PIC) modeling in addition to hybrid simulations that can cover a longer time period and have been successfully employed for oblique nonrelativistic shocks (e.g., Gargaté \& Spitkovsky 2012). 
We have embarked on very large scale kinetic plasma simulations of the formation of unmagnetized or weakly magnetized strictly parallel $\left(\theta_{\mathrm{Bn}}=0^{\circ}\right)$ nonrelativistic shocks, their longterm evolution, and particle acceleration. To address the question whether or not the shock structure and the particle spectra ever reach a steady state, we follow the evolution of the system longer than was done in Kato \& Takabe (2008). We are interested in asymmetric flows, i.e., the collision of plasma slabs of different density, leading to two different shocks and a contact discontinuity (CD). The Mach number is set high enough that electrostatic shocks will not be formed. We want to see whether a parallel magnetic field renders shock formation more efficiently. In the simulations with magnetized plasma, the magnetic field strength is assumed sufficiently low for the formation of the forward and reverse shocks with large Alfvénic Mach number, $M_{\mathrm{A}} \gg 1$. This, together with the assumption of sub-relativistic plasma collision speed, renders our simulations applicable to the conditions at young SNRs.

In contrast to a conducting-wall reflection method to set up the shock, used in Kato \& Takabe (2008), our asymmetric slabcollision model is physically more accurate as it avoids assuming the existence of an infinitely sharp CD (in fact a grid-cell wide) and allows us to properly investigate the dynamics of the forward and reverse shocks for different stream-counterstream parameters and magnetic field configurations. Beam-plasma systems are susceptible to various instabilities: some electrostatic (e.g., two-stream or Buneman modes), other quasi-electrostatic (e.g., Bret et al. 2010), and still others electromagnetic (e.g., filamentation). The unstable spectrum is thus at least two-dimensional. Which of these modes would grow fastest highly depends on the system parameters (Bret 2009). This clearly demonstrates a need for studies using a setup like ours, since the most unstable modes excited in various settings may result in completely different shock-generating plasma waves.

Besides demonstrating the efficiency of the shock-formation processes in the presence of the mean magnetic field, a secondary advantage of restricting to a parallel or absent largescale magnetic field is that simulations can be set up cleaner. PIC simulations are sensitive to electromagnetic transients that arise from the initial setup and propagate through the simulation box. In shock simulations they can induce scattering turbulence and electron heating in the upstream region of the shock. Likewise, a perpendicular component of the homogeneous magnetic field implies that in the collision region (or reflecting plane, if that is used to set up the shock) the curl of either the magnetic field or the motional electric field is large, effectively acting as an antenna and emitting an electromagnetic pulse.

The simulation model and setup are described in Section 2. The results of the simulations are presented in Section 3. We conclude with a summary and discussion in Section 4.

\section{SIMULATION SETUP}

In our simulations, two electron-ion plasma streams of different densities collide with each other at relative speed $v_{\text {rel }}=0.38 c$. The density ratio between the dense and the tenuous-plasma slab is 10 . The simulation frame of reference is the center-of-momentum frame of the two plasmas, in which the dense (left) plasma stream moves to the right in $+x$-direction with velocity $\mathbf{v}_{\mathrm{L}}=0.0354 c \hat{\mathbf{x}}$ and the tenuous (right) stream moves to the left in $-x$-direction at $\mathbf{v}_{\mathrm{R}}=$ $-0.354 c \hat{\mathbf{x}}$. For simplicity, we will refer to the electrons and ions of the dense-plasma stream as dense electrons and dense ions, and as tenuous electrons and tenuous ions for the dilute plasma. The plasmas are initially very cold; in the respective rest frames of the streams, the electrons have a thermal distribution with $v_{e, \text { th }}=\sqrt{k T / m_{e}}=0.002 c$ and are in thermal equilibrium with the ions. We consider both unmagnetized and magnetized plasma conditions. In the latter case, the plasma flow is aligned with the homogeneous magnetic field, $B_{0, x}$, and thus we restrict our study to the formation of parallel shocks. The mean magnetic field strength is given by the ratio of the electron cyclotron frequency $\Omega_{e}=e B_{0, x} / m_{e}$ to the electron plasma frequency of the dense-plasma component, $\omega_{\mathrm{pe}, L}=\sqrt{e^{2} N_{e, L} / \epsilon_{0} m_{e}}$ (where $\epsilon_{0}$ is the vacuum permittivity and $N_{e, L}$ is the density of dense electrons), which is $\Omega_{e} / \omega_{\mathrm{pe}, L}=0.04$. Such a flowaligned magnetic field allows filamentation-like instabilities. As shown in, e.g., Bret et al. (2006), for the parameters of the initial plasma slab collision considered here and taking into account counterstreaming electron beams only, a plasma magnetization larger than $\Omega_{e} / \omega_{\mathrm{pe}, L} \simeq \beta_{\text {rel }} \sqrt{0.1 \gamma_{\text {rel }}} \approx 0.125$ would be required to suppress purely transverse filamentation modes, whereas a complete stabilization of oblique modes would never be efficient. With the assumed plasma magnetization of one-third of the suppression field, the filamentation instability growth rate is reduced by $10 \%$ only. The simulation setup is thus designed to be applicable to young SNRs, in which dense supernova ejecta propagating with nonrelativistic velocity collide with a dilute weakly magnetized interstellar medium.

On account of the slower rate of shock-forming instabilities in nonrelativistic flows, our large-scale simulations of nonrelativistic plasma collisions have been performed in two spatial dimensions but keeping three components of the particle velocities (a $2 \mathrm{D} 3 \mathrm{~V}$ model). The code used in this study is a $2 \mathrm{D} 3 \mathrm{~V}$ modified version of the relativistic electromagnetic particle code TRISTAN with MPI-based parallelization (Buneman 1993; Niemiec et al. 2008). We have performed extensive test simulations to formulate a numerical model that best conserves energy and minimizes numerical self-heating to provide a coldplasma beam that is sufficiently stable against numerical gridCerenkov and other instabilities. The model takes advantage of second-order particle shapes and uses a second-order FDTD field-solver with a weak Friedman filter (Greenwood et al. 2004) to suppress small-scale noise. In comparison with the numerical model used in previous work (e.g., Niemiec et al. 2008; Stroman et al. 2012), that employed first-order particle shapes and a computationally expensive iterative algorithm for digital filtering of electric currents, the current model allows to maintain a satisfactorily low noise level with fewer particles per cell, thus providing an increased overall performance of the code (compare Fonseca et al. 2008).

Table 1 summarizes the parameters of the main simulation runs discussed in this study. We use five particles per cell for each of the four plasma species in the active grid and apply the splitting method for the tenuous plasma, which assigns statistical weights to each particle to maintain the desired density ratio. Thus, each ion particle can be initialized at the same location as the corresponding electron, to ensure a vanishing initial charge density. The electron skin depth of the dense-plasma component $\lambda_{\mathrm{se}, L}=c / \omega_{\mathrm{pe}, L} \simeq 8.1 \Delta$, where $\Delta$ is the grid-cell size. Because the characteristic length scales of the plasma ions should be well contained in the simulation box, we cannot use a realistic proton-to-electron mass ratio. Here we base our discussion on the results obtained for a reduced ion-electron mass ratio $m_{i} / m_{e}=50$ (runs U1 and M1 in Table 1). A slight dependence of the simulation results on the assumed mass ratio has been 
Table 1

Parameters of the Main Simulation Runs

\begin{tabular}{lcccc}
\hline \hline Runs & $m_{i} / m_{e}$ & $\Omega_{e} / \omega_{\mathrm{pe}, L}$ & $\begin{array}{c}\text { Grid } \\
\left(\lambda_{\mathrm{si}}^{2}\right)\end{array}$ & $\begin{array}{c}t^{\mathrm{max}} \\
\left(\omega_{\mathrm{pi}}^{-1}\right)\end{array}$ \\
\hline $\mathrm{U} 1$ & 50 & 0 & $6122.5 \times 37.8$ & 4111 \\
$\mathrm{U} 2$ & 20 & 0 & $5531.7 \times 39.8$ & 3803 \\
M1 & 50 & 0.04 & $6122.5 \times 37.8$ & 4111 \\
M2 & 20 & 0.04 & $5531.7 \times 39.8$ & 3803 \\
\hline
\end{tabular}

Notes. Parameters of the main simulation runs described in this paper. Listed are the ion-electron mass ratio $m_{i} / m_{e}$, plasma magnetization, maximum computational box size in units of the ion skin depth of the dense plasma ( $\lambda_{\mathrm{si}}=57.2$ for runs $\mathrm{U} 1$ and M1 with $m_{i} / m_{e}=50$ and $\lambda_{\mathrm{si}}=36.2$ for runs U2 and M2 with $m_{i} / m_{e}=20$ ), and the run duration in units of $\omega_{\mathrm{pi}}^{-1}$.

observed in Kato \& Takabe (2008). To ascertain the effects of this parameter on the long-time evolution of the system, we performed large-scale simulations also for $m_{i} / m_{e}=20$ (runs $\mathrm{U} 2$ and M2); the results of these simulations are summarized in Appendix A. For runs U1 and M1, referred to here as the unmagnetized and magnetized simulation, respectively, the ion skin depth of the dense plasma is $\lambda_{\mathrm{si}} \equiv \lambda_{\mathrm{si}, L}=c / \omega_{\mathrm{pi}, L} \simeq 57.2 \Delta$. Hereafter, $\lambda_{\text {si }}$ will be used as the unit of length, and we take the inverted ion plasma frequency of the dense component, $\omega_{\mathrm{pi}}^{-1} \equiv \omega_{\mathrm{pi}, L}^{-1}$, as our unit of time. The time step we use is $\delta t=0.062 \omega_{\mathrm{pe}, L}^{-1}=0.0087 \omega_{\mathrm{pi}}^{-1}$.

The simulations have been performed on a spatial grid in the $x-y$ plane with radiating boundary conditions in $x$ and periodic boundary conditions in $y$. The transverse size of the computational grid used in runs $\mathrm{U} 1$ and M1 is $L_{y}=37.8 \lambda_{\mathrm{si}}$, and the system evolution has been followed for $4111 \omega_{\mathrm{pi}}^{-1}$, i.e., $\sim 2.9 \times 10^{4} \omega_{\mathrm{pe}, L}^{-1}$, which in simulation M1 corresponds to $23.5 \Omega_{i}^{-1}$, where $\Omega_{i}$ is the ion cyclotron frequency. The simulations thus require a very large grid size in plasma flow direction, because the interpenetrating plasma streams occupy a continuously increasing volume. Our MPI-parallelized model partitions the simulation box into processor domains of equal size, and the number of processor domains in the transverse direction is constant. To limit the computational expense, in terms of both processor usage and memory, an actual simulation is broken into several segments of different size in $x$, for which the number of processor domains in flow direction is increased. Thus, to complete a simulation with, e.g., 200,000 time steps, succeeding runs with one, two, four, and six 216processor domains each with $25,000 \times 10$ cells are performed on 216, 432, 864, and 1296 cores, respectively. Particles are continuously added to the system on both sides of the collision region with a so-called moving injector. In this method, the injection layer moves away from the interaction region at a distance that preserves all the particles and fields generated in the collision region, but at the same time it does not allow a beam to travel a long distance without interaction with counterstreaming particles, which further helps avoid numerical grid-Cerenkov effects.

Note that the parameters used in the main numerical experiments were chosen following an extended scan of the parameter space (e.g., number of particles per cell, the electron skin depth, the magnetic field strength) performed with smaller-size simulations. The results of the two selected production runs, U1 and M1, are thus representative of the physical effects observed in the unmagnetized and weakly magnetized system. The validity of the two-dimensional simulations in capturing the essential physics of parallel shock formation through the electromagnetic instabilities has been demonstrated in numerous studies of relativistic and nonrelativistic shocks (e.g., Spitkovsky 2008; Niemiec et al. 2008; Kato \& Takabe 2008).

The collision of the two plasma streams, as studied in this work, should in the nonlinear stage result in the formation of a system of forward and reverse shocks separated by a CD. The shock structures observed in our simulations can thus be compared with the predictions of a hydrodynamic model. In analogy to the analysis presented in Nishikawa et al. (2009), we can derive the CD speed, which in the simulation frame is $\boldsymbol{\beta}_{\mathrm{CD}}=-0.06 \hat{\mathbf{x}}$. The CD moves because the system is initially under momentum balance but not ram-pressure balance. The velocities of the forward and reverse shocks depend on the adiabatic index of the shocked plasma, which is different for unmagnetized and magnetized plasmas in the simulations. In the unmagnetized case, particle motion is confined to the simulation plane, and the nonrelativistic adiabatic index is $\Gamma_{\mathrm{U}}=2$. In the magnetized case, the magnetic field bends the particle trajectories out of the simulation plane. In effect, particles have three degrees of freedom, and the nonrelativistic adiabatic index is $\Gamma_{M}=5 / 3$. In the following we use subscripts " $U$ " and " $M$ " to denote quantities measured in the unmagnetized and magnetized run, respectively. Subscripts "R" and "L" refer to the rest frame of the tenuous and dense plasma, respectively. These subscripts are dropped for quantities measured in the simulation frame.

For an unmagnetized-plasma collision, the steady-state speed of the forward and the reverse shock should approach $\beta_{\mathrm{fR}, \mathrm{U}}=$ 0.43 and $\beta_{\mathrm{rL}, \mathrm{U}}=-0.14$, in the rest frame of the tenuous and dense beam, respectively. In the simulation frame, the shock velocities are $\beta_{\mathrm{f}, \mathrm{U}}=0.09$ and $\beta_{\mathrm{r}, \mathrm{U}}=-0.11$. The compression ratios at each shock are predicted to be $N_{\mathrm{fU}, \mathrm{d}} / N_{\mathrm{fU}, \mathrm{u}}=2.9$ and $N_{\mathrm{rU}, \mathrm{d}} / N_{\mathrm{rU}, \mathrm{u}}=3.01$. In the magnetized case, the simulationframe velocities of the forward and the reverse shocks should be $\beta_{\mathrm{f}, \mathrm{M}}=0.04$ and $\beta_{\mathrm{r}, \mathrm{M}}=-0.09$, and the shock compression ratios $N_{\mathrm{fM}, \mathrm{d}} / N_{\mathrm{fM}, \mathrm{u}}=3.86$ and $N_{\mathrm{rM}, \mathrm{d}} / N_{\mathrm{rM}, \mathrm{u}}=4.02$, respectively. The Alfvén velocity in the dense plasma is $v_{\mathrm{A}, \mathrm{L}}=$ $\left[B_{0, x}^{2} / \mu_{0}\left(N_{e, L} m_{e}+N_{i, L} m_{i}\right)\right]^{1 / 2}=5.67 \times 10^{-3} c$. In the rest frame of the tenuous plasma, the Alfvén speed $v_{\mathrm{A}, \mathrm{R}}=1.85 \times 10^{-2} c$. In the upstream rest frames of the tenuous and dense plasma the velocities of the forward and reverse shocks read $\beta_{\mathrm{fR}, \mathrm{M}}=0.39$ and $\beta_{\mathrm{rL}, \mathrm{M}}=-0.13$, respectively. Thus, the Alfvénic Mach number of the forward shock is $M_{\mathrm{Af}}=c \beta_{\mathrm{fR}, \mathrm{M}} / v_{\mathrm{A}, \mathrm{R}} \simeq 21.1$ and that of the reverse shock is $M_{\mathrm{Ar}}=c \beta_{\mathrm{rL}, \mathrm{M}} / v_{\mathrm{A}, \mathrm{L}} \simeq 22$. . $^{7}$

\section{SIMULATION RESULTS}

In what follows, we present the results of our two main numerical experiments for unmagnetized-plasma conditions (run U1, Section 3.1) and for magnetized plasma (run M1, Section 3.2), both of which use an ion-electron mass ratio $m_{i} / m_{e}=50$. The results of two other large-scale runs, U2 and M2, which assume a lower-mass ratio $m_{i} / m_{e}=20$, are described in Appendix A. The analysis of simulation data is also supported with additional test runs.

To understand the nature of various unstable wave modes that appear in the systems, we also perform a linear analysis. Given the complexity of the situation and the need to investigate the full spectrum of unstable waves, our linear calculations are restricted to the cold-plasma regime. We thus write the cold fluid equations for each of the plasma components and derive the

\footnotetext{
7 Customarily stated upstream flow Mach numbers read $M_{\mathrm{Af}}=v_{\mathrm{R}} / v_{\mathrm{A}, \mathrm{R}} \simeq 19.1$ and $M_{\mathrm{Ar}}=v_{\mathrm{L}} / v_{\mathrm{A}, \mathrm{L}} \simeq 6.3$.
} 
dielectric tensor $\mathbf{T}(\mathbf{k}, \omega)$ adapting the Mathematica Notebook described in Bret (2007). For both unmagnetized plasma and a flow-aligned magnetic field, we can without loss of generality restrict the wavevector to the $x-y$ plane, $\mathbf{k}=\left(k_{x}, k_{y}\right)$. The dispersion relation then reads $\operatorname{det} \mathbf{T}(\mathbf{k}, \omega)=0$, from which we calculate the growth rates of unstable wave modes.

\subsection{Unmagnetized Plasma}

\subsubsection{Early-stage Evolution}

In the first stage of the plasma collision, the interpenetration of dense and tenuous streams quickly leads to current filamentation and the generation of transverse (out of plane in our twodimensional setup) magnetic field in the collision region. The observed turbulent modes result from Weibel-like instabilities, and their spectrum is dominated by modes with wavevectors oriented obliquely to the plasma flow (Bret et al. 2005). This is in agreement with our linear analysis in Stroman et al. (2012) that assumed similar stream-counterstream relative velocity and density ratio. The amplified magnetic field influences the motion of particles. The ion beams slightly decelerate in bulk and develop a population of slow particles. At the same time the dilute-ion beam, in which filamentation is strongest, is heated. The bulk kinetic energy released by the decelerating ions is converted to electron heating in the electrostatic fields that accompany the ion filaments (e.g., Hededal et al. 2004; Medvedev 2006; Nishikawa et al. 2006; Spitkovsky 2008). A fraction of the electrons of both streams is reflected away from the collision region. The initially reflected electrons of the tenuous plasma constitute a distinct hot beam that counterstreams against the incoming particles of the tenuous stream.

By the time $t \sim 400 \omega_{\mathrm{pi}}^{-1}$, the magnetic turbulence has reached sufficient strength that fresh incoming upstream electrons can no longer cross the collision region. A CD is formed that separates the electrons of both plasma slabs that slowly accumulate at this barrier. The structure of the collision region at this early evolution stage is shown in Figure 1 . The CD is located around the initial collision boundary at $x / \lambda_{\mathrm{si}} \approx 3120$. As shown by the average-density profiles (Figure 1(a)) and particle density distributions (Figures $1(\mathrm{~d})-(\mathrm{g})$ ), the CD creates a barrier for less energetic dense ions. Only a few of these particles penetrate the tenuous-plasma region, where they help maintain charge neutrality. Since the number density of the dense ions in the tenuous-plasma region is comparable to that of the tenuous ions, the former play an important dynamical role in the system. The tenuous ions are only weakly perturbed upon crossing the CD. The fast tenuous-ion beam propagates into the extended region to the left of the CD. Its net charge is compensated in part by electrons of the dense beam that have been reflected during the initial plasma encounter and in part by hot tenuous electrons that leak across the CD. Very few dense electrons cross the CD into the tenuous-plasma region. In this zone, the charge balance with the dense ions streaming against the dilute beam is provided by reflected tenuous electrons.

The structure of magnetic turbulence shows different characteristics on the two sides of the CD (Figure 1(c)). In the denseplasma region, the tenuous fast beam constitutes a perturbation to the system. Ion-ion counterstreaming in this asymmetric beam/plasma system renders the unmagnetized oblique instability dominant in the spectrum of unstable modes. Obliquefilamentary structures of wavelength $\lambda \sim 3.5 \lambda_{\text {si }}$ can be seen in the transverse component of the magnetic field and also
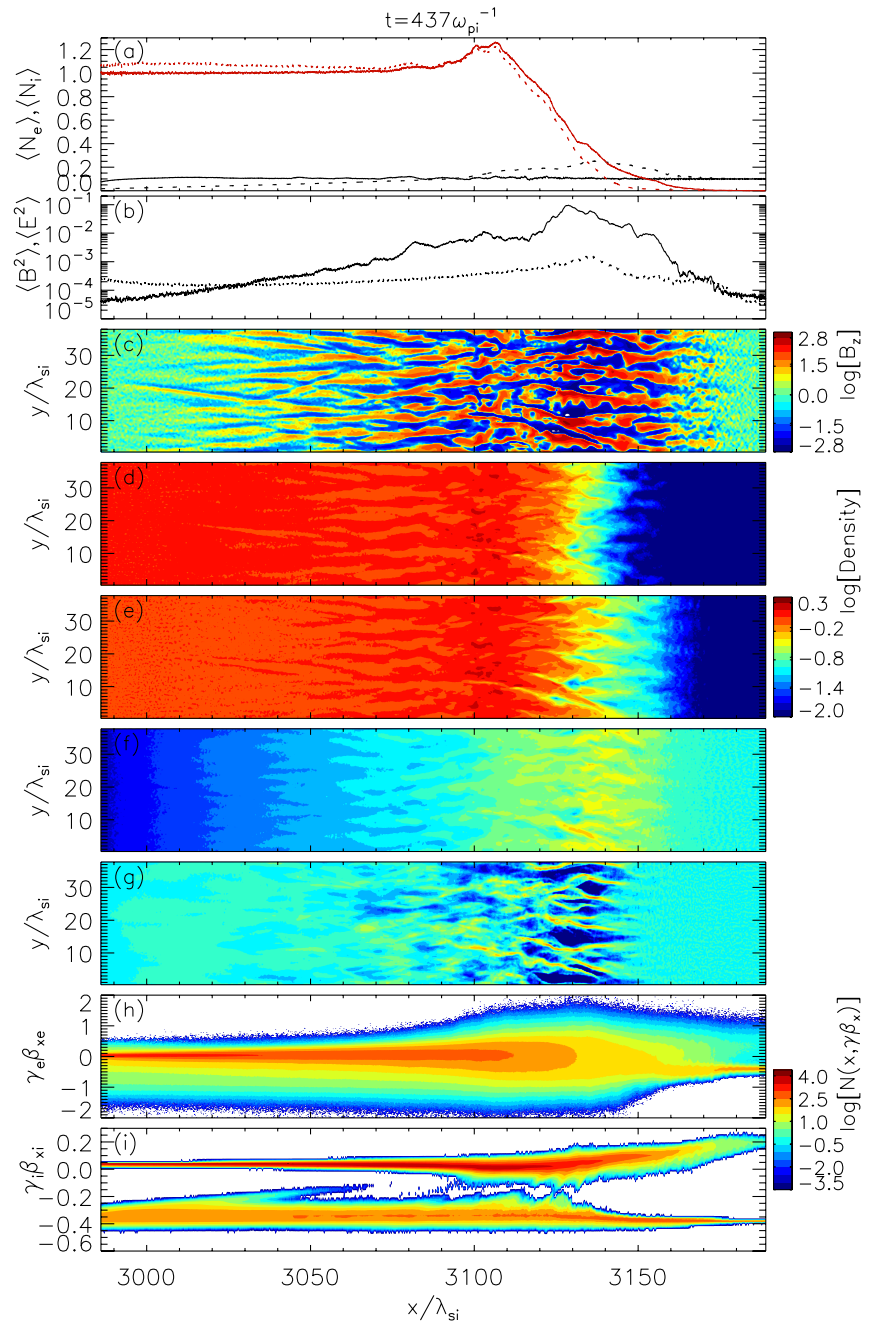

Figure 1. Structure of the plasma-collision region at time $t=437 \omega_{\mathrm{pi}}^{-1}$ for the unmagnetized run U1. Displayed are the profiles of (a) the average particlenumber density normalized to the far-upstream density of the dense plasma (red lines: dense plasma, black lines: tenuous plasma; solid lines: ions, dotted lines: electrons), (b) the average magnetic (solid line) and electric (dotted line) energy density in simulation units, (c) the amplitude of the magnetic field $B_{z}$ (in sign-preserving logarithmic scale as $\left.\operatorname{sgn}\left(B_{z}\right)\left(3+\log \left[\max \left(10^{-3},\left|B_{z}\right|\right)\right]\right)\right)$, the density of dense electrons (d), dense ions (e), tenuous electrons (f), and tenuous ions $(\mathrm{g})$, all normalized to the far-upstream density of the dense plasma, and the longitudinal phase-space distribution of electrons (h) and ions (i).

(A color version of this figure is available in the online journal.)

through density modulations of the electrons and ions (Stroman et al. 2012). The density filaments are stronger in the tenuous plasma because they can be compensated by much weaker perturbations of the dense beam. Weak-beam conditions are also found at the leading edge of the dense-ion beam propagating into the tenuous-plasma region to the right of the CD. Here, the dilute beam is composed of the most energetic ions of the dense slab that have been accelerated in the collision region (Figure 1(i)). The oblique mode is thus excited with wavelength $\lambda \sim 5 \lambda_{\text {si }}$, comparable to the skin depth of the tenuousion component. However, the dense-ion beam shows a very strong density gradient in the tenuous-plasma region. The conditions that determine the spectrum of unstable modes thus vary with distance from the $\mathrm{CD}$ from ones suitable for the oblique mode farther upstream (at $x / \lambda_{\mathrm{si}} \approx 3170$ ) to those in the region closer to the $\mathrm{CD}$ (at $x / \lambda_{\mathrm{si}} \approx 3150$ ) that allow quasiperpendicular filamentation-like modes $\left(k_{\|} \approx 0\right)$ to grow fastest. In effect, the magnetic structure formed at the dilute head of the 


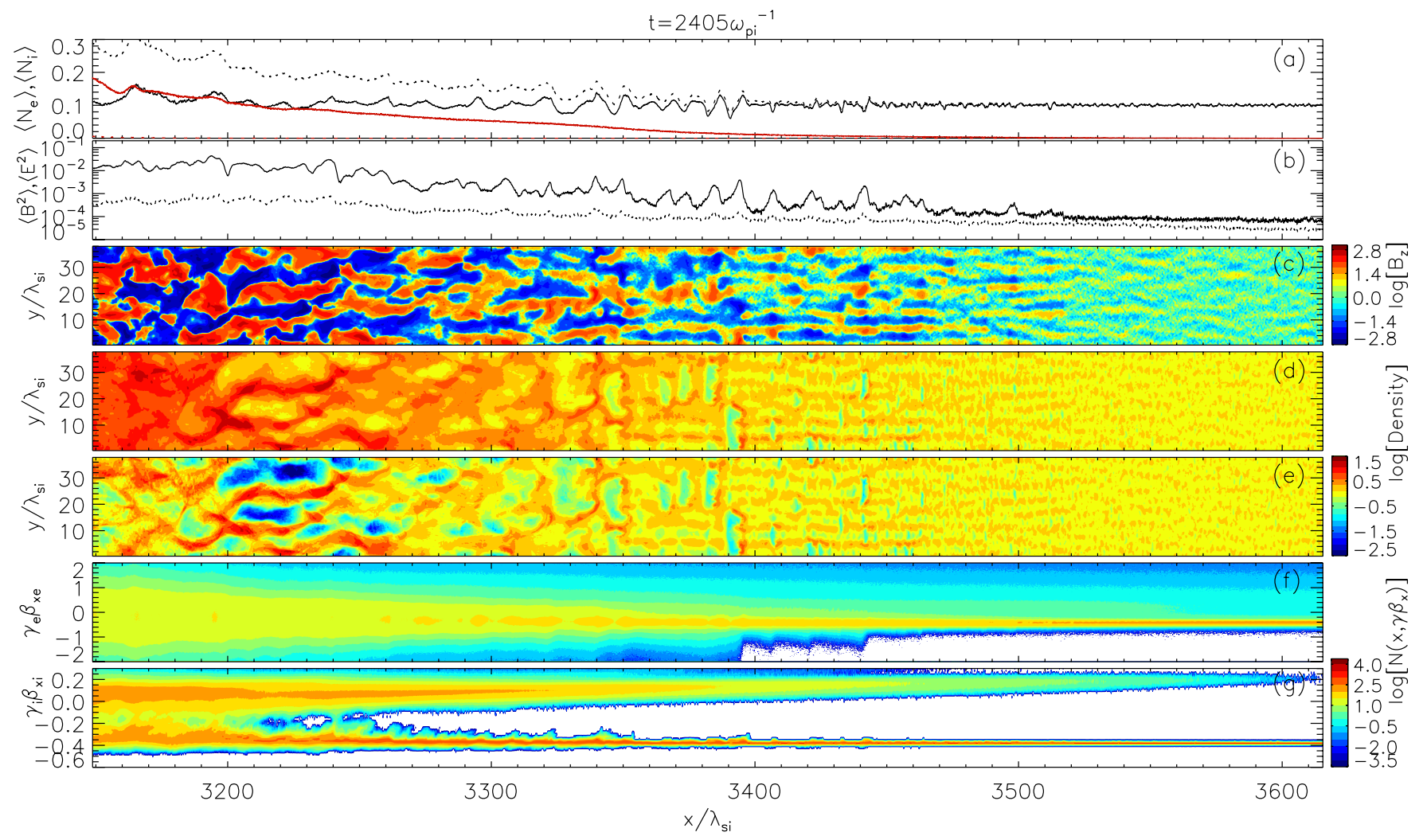

Figure 2. Structure of the forward-shock precursor at time $t=2405 \omega_{\mathrm{pi}}^{-1}$. Shown are the profiles of (a) the average particle number density, (b) the average magnetic and electric energy density (see Figures 1 (a) and (b)), (c) amplitude of magnetic field $B_{z}$ (logarithmic scale, see Figure 1(c)), the density of tenuous electrons (d), tenuous ions (e), both normalized to the far-upstream density of the tenuous plasma, and the longitudinal phase-space distribution of electrons (f) and ions (g).

(A color version of this figure is available in the online journal.)

dense-ion beam becomes strongly modified by a filamentationlike instability that grows faster than the oblique instability and therefore leads to more efficient magnetic field amplification. As seen in Figure 1, the instabilities spatially separate the ions of both slabs. Narrow filaments in cold tenuous ions are surrounded by electrons. The volume between the filaments is depleted of tenuous ions and occupied by the ions of the dense beam. The filamentary structures in the electron components are more diffuse on account of the higher temperature of these particles. The turbulent magnetic structure advected toward the $\mathrm{CD}$ is further amplified through mergers of current filaments. The observed characteristics of the magnetic field generation in the tenuous-plasma region are thus reminiscent of the Weibelmediated relativistic shocks in unmagnetized plasmas (e.g., Medvedev \& Loeb 1999; Silva et al. 2003; Nishikawa et al. 2003; Frederiksen et al. 2004).

Whereas the filamentation instability operating in the tenuous plasma can generate strong magnetic turbulence in this region, the oblique instability leads to only moderate magnetic field amplification in the dense plasma left of the CD. As shown in Figure 1(b), the energy density of the magnetic field is more than an order of magnitude larger in the region to the right of the $\mathrm{CD}$, and this proportion is not significantly modified during the subsequent evolution of the system. The strong magnetic field generated in the $\mathrm{CD}$ region starts to efficiently deflect and isotropize particles. The flow speed of the bulk plasma decreases, and larger numbers of ions are reflected back toward their respective upstream regions (Figure 1(i)). The bulk kinetic energy of decelerating ions is converted into electron heating (Figure 1(h)). These processes of energy dissipation should enforce the formation of a pair of shocks on the two sides of the CD.

\subsubsection{Late-stage Evolution of the Forward-shock Transition}

In the tenuous-plasma region, at $t \sim 500 \omega_{\mathrm{pi}}^{-1}$ the compression ratio reaches the level of $\sim 3$ predicted by the hydrodynamic jump conditions for a two-dimensional nonrelativistic plasma with adiabatic index $\Gamma_{\mathrm{U}}=2$. The compression of electrons is provided solely by the tenuous-electron component, whereas the ion compression is initially dominated by the dense ions (see Figure 2). The extended region between the unperturbed dilute plasma and the $\mathrm{CD}$ constitutes a forming forward shock.

The structure of the forward-shock precursor (in the right half of the simulation box) is initially exclusively controlled by strongly nonlinear interactions between counterstreaming ion beams resulting in the formation and mergers of current filaments (see, e.g., Medvedev et al. 2005; Polomarov et al. 2008; Shvets et al. 2009). The corresponding turbulence is not purely magnetic and transverse. The two ion beams pinch at a different rate, on account of their different density and temperature. Space charges thus arise that induce a weak in-plane electric field, $E_{y}$, which is not shown here (Tzoufras et al. 2006; Fiore et al. 2006). In the nonlinear phase, the magnetic-pressure gradient can also drive strong electrostatic fields (Dieckmann et al. 2009). The size of the magnetic precursor is thus determined by the range of the dense-ion beam in the tenuous plasma. Ahead of the filaments, a population of hot tenuous electrons, which have been reflected in the $\mathrm{CD}$ region, streams with mildly relativistic velocity against the incoming beam. This excites a two-stream 
instability between reflected electrons and beam electrons that produces mainly longitudinal $\left(\mathbf{k} \times \mathbf{v}_{\mathrm{R}}=0\right)$ perturbations in $E_{x}$ and associated electron charge-density modulations with wavelength $\lambda \sim 5.5 \lambda_{\mathrm{se}, R}$, in agreement with our linear analysis. ${ }^{8}$ The amplitude of electrostatic perturbations saturates at small level, and their main effect is to heat up the electrons of the incoming beam far upstream of the forward shock. However, deeper in the forward-shock precursor the electron-density perturbations become nonlinearly enhanced through the approaching stream of the dense ions, and the fluctuations in $E_{x}$ are amplified to amplitudes far exceeding those farther upstream. This leads to a strongly nonlinear backreaction on the tenuous-plasma ions, whose density structure begins to resemble that of the electrons. Figure 2 presents the structure of the precursor region at time $t=2405 \omega_{\mathrm{pi}}^{-1}$ that is representative of the characteristics discussed above. The figure shows profiles of the average particle densities (Figure 2(a)) and electromagnetic-energy densities (Figure 2(b)), a two-dimensional distribution of the magnetic field $B_{z}$ (Figure 2(c)), the densities of electrons and ions of the tenuous plasma (Figures 2(d) and (e)), and the electron and ion phase-space density (Figures 2(f) and (g)). The electrons of the dense plasma are absent in this region. The density of the denseion beam is homogeneous and shows a strong gradient (see red solid line in Figure 2(a) and the beam with positive momentum in Figure $2(\mathrm{~g}))$. The far-upstream region is excluded from Figure 2, but the hot tenuous electrons counterstreaming against the incoming beam can be clearly seen as a population with positive momenta beyond $x / \lambda_{\mathrm{si}} \approx 3550$ in Figure 2(f). The strong density fluctuations in the ions and electrons of the tenuous plasma can be seen in Figures 2(d) and (e) for $x / \lambda_{\text {si }} \gtrsim 3450$. The longitudinal structures coexist with transverse density filaments, indicating that the filamentation-like instability and the density modulation discussed above operate in parallel. Closer to the shock, the density modulations eventually lead to the formation of plasma cavities. In this region, the density of the dense-ion beam is increased and also a population of reflected tenuous ions exists (for $x / \lambda_{\text {si }} \lesssim 3400$; the density of the shock-reflected ions is more than a factor of 10 smaller than that of the dense ions). Such nonlinear structures are known to frequently occur in plasma physics and astrophysics applications (e.g., Goldman 1984). As the parallel density structures are advected toward the shock and the density of the dense-ion beam/shock-reflected ions increases, the modulations grow further in amplitude and eventually begin to rapidly expand radially. The expansion and subsequent merging of the adjacent cavities cause a compression of the plasma between the voids and enhance electric currents in the compression regions that lead to additional magnetic field amplification.

The filamentary structure of the magnetic field thus becomes modified in the shock precursor. This can be observed in Figure 2(c) and in the mean magnetic-energy-density profile in Figure 2(b) (solid line), in which the effects discussed are revealed as localized magnetic field amplification. Note that although the interaction between the dense-ion beam and the charge-density waves turns highly nonlinear, it is the filamentation instability that remains the dominant source of mag-

\footnotetext{
8 The linear analysis performed for conditions representative of the far-upstream forward-shock precursor also shows the existence of a weak filamentation mode that is in fact observed but does not provide a significant dynamical impact on the precursor. The third predicted mode-the Buneman mode resulting from the drift of electrons against the ions, which is needed for current balance with the reflected electrons, is an artifact of the zero-temperature limit assumed in the calculations and should dissipate quickly in the warm precursor plasma.
}

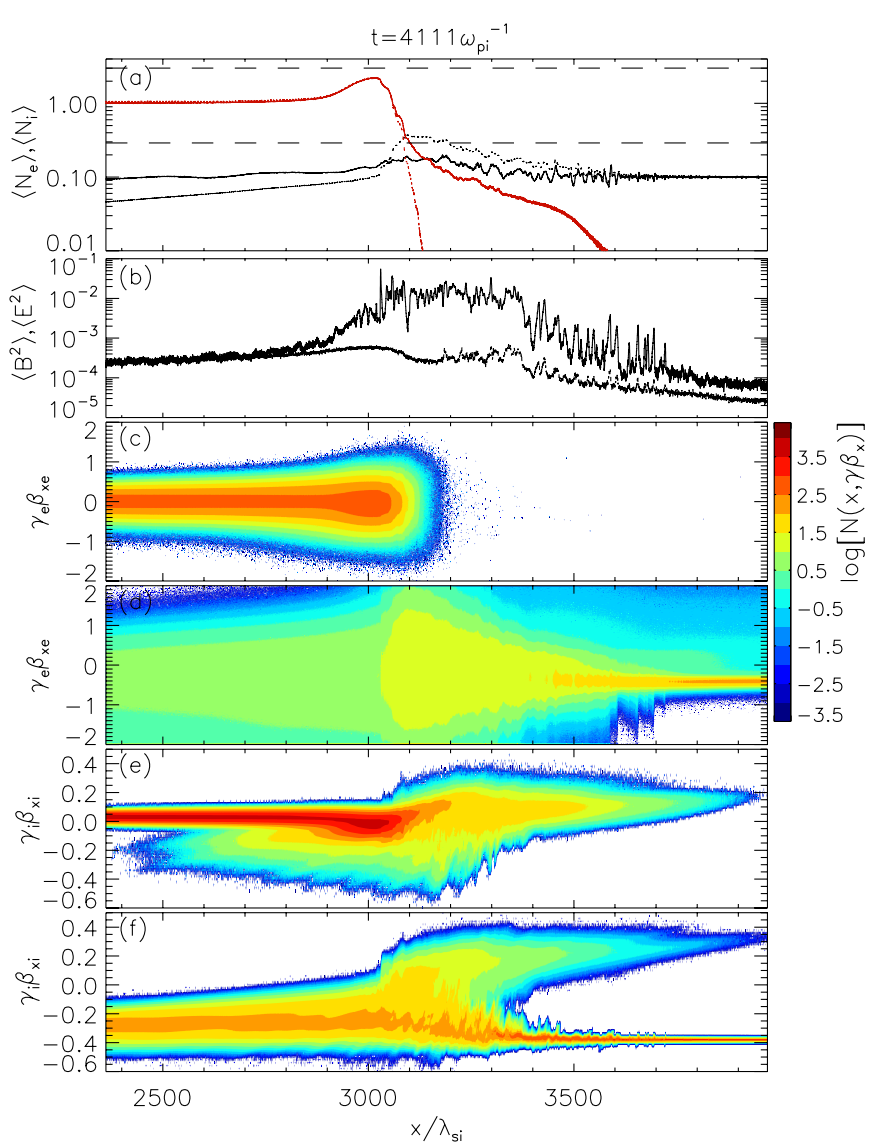

Figure 3. Structure of the collision region at the end of the simulation U1 at time $t=4111 \omega_{\mathrm{pi}}^{-1}$. Shown are the profiles of (a) the average particle-number density (a logarithmic scale is used), (b) the average magnetic and electric energy density (see Figures 1(a) and (b)), and the longitudinal phase-space distributions separately for all particle species: electrons of the dense (c) and tenuous (d) plasma and ions of the dense (e) and tenuous (f) component. In panel (a), the horizontal dashed lines mark the hydrodynamic compression level of $N_{\mathrm{fU}, \mathrm{d}} / N_{\mathrm{fU}, \mathrm{u}}=2.9$ for the forward shock (lower line) and $N_{\mathrm{rU}, \mathrm{d}} / N_{\mathrm{rU}, \mathrm{u}}=3.01$ for the reverse shock (upper line).

(A color version of this figure is available in the online journal.)

netic field generation. As is evident in Figure 2(b), the average magnetic-energy density in the region of nonlinear filamentation $\left(x / \lambda_{\text {si }} \lesssim 3280\right)$ is about an order of magnitude larger than the energy density associated with plasma compressions farther upstream. Nevertheless, the role of density modulations is not negligible. It is also important to note that the volume filled with weak electrostatic turbulence ahead of the magneticfilamentation zone constitutes an inherent part of the shock precursor and as such must be included in realistic PIC modeling. The electrostatic precursor is not a transient effect in nonrelativistic shocks because there will always be a population of electrons reflected upstream with relativistic velocities that outrun the bulk of the shock-reflected ions.

In our symmetric unmagnetized test run (equal density in the colliding plasma slabs) we see similar effects in the shock precursor, but the cavities represent weaker density fluctuations and do not grow nonlinearly as in the asymmetric simulation U1. Consequently, magnetic field filaments are only weakly modified by plasma compression. The cavities that form upstream dissipate closer to the shock and give place to density filaments.

Figure 3 shows the structure of the collision region at the end of the simulation at time $t=4111 \omega_{\mathrm{pi}}^{-1}$. The profiles of the average particle densities (Figure 3(a)) and electromagnetic- 


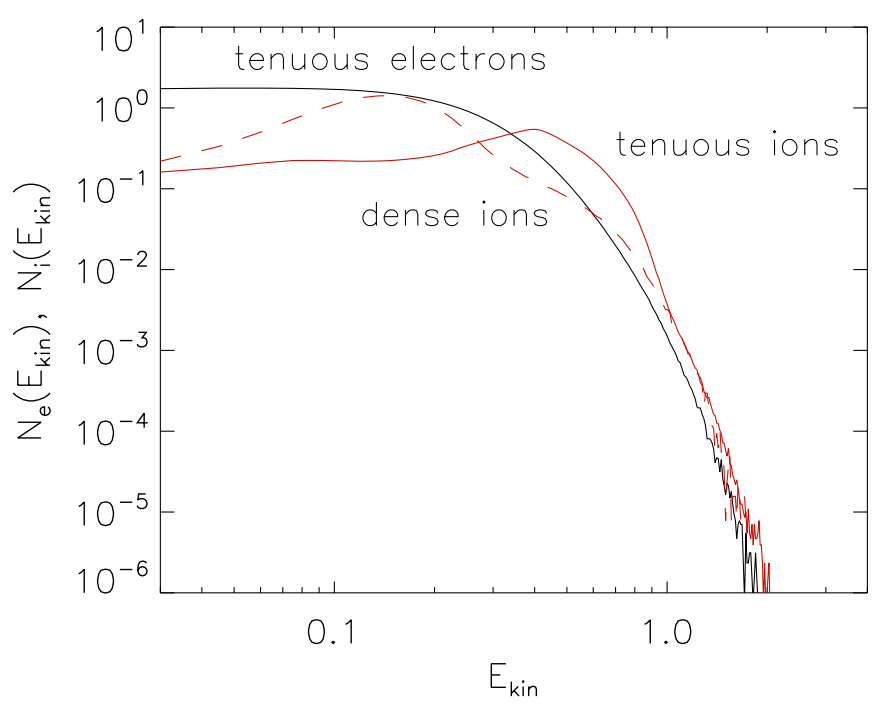

Figure 4. Kinetic-energy spectra of particles downstream of the forward shock at $x / \lambda_{\mathrm{si}} \approx 3120$ and time $t=4111 \omega_{\mathrm{pi}}^{-1}$ for the unmagnetized run $\mathrm{U} 1$, in the CD rest frame. In simulation units $E_{\text {kin }}=0.25(\gamma-1)$ for electrons and $E_{\text {kin }}=12.5(\gamma-1)$ for ions. Normalized spectra are shown for tenuous electrons (black solid line), tenuous ions (red solid line), and the dense ions (red dashed line).

(A color version of this figure is available in the online journal.)

energy densities (Figure 3(b)) are presented together with the phase-space distribution separately for all particle species (Figures 3(c)-(f)). The plasma density and electromagnetic field structure (not shown) are analogous to that at the earlier stage as described above (compare Figures 2(a) and (b) with Figures 3(a) and $(b))$. The CD is located at $x / \lambda_{\mathrm{si}} \approx 3050$. By the end of the run, its speed approaches the predicted $\beta_{\mathrm{CD}}=-0.06$ in the simulation frame. The forward-shock region extends to the right of the $\mathrm{CD}$. The shock compression is in agreement with a hydrodynamic value of $N_{\mathrm{fU}, \mathrm{d}} / N_{\mathrm{fU}, \mathrm{u}}=2.9$ (lower dashed line in Figure 3(a)), but the shock front is poorly defined.

The plasma filaments that grow in size when approaching the forward shock should disintegrate to form a nearly homogeneous downstream region. However, this stage is not yet observed by the end of the simulation. Although the downstream tenuous-ion density distribution is strongly turbulent, it is evident from Figure 3(f) that the dilute-ion beam is not decoupled from the dense plasma and, despite being hot, continues to carry a substantial bulk-flow energy across the CD. Consequently, the forward-shock compression ratio carries a substantial contribution from the dense ions. Note in Figure 3(a) that the density of dense ions, roughly equal to the tenuous-ion density, is required to match the electron compression that is provided solely by tenuous electrons. As shown in Figure 4, which displays particle distributions in the forward-shock downstream, the tenuous-ion spectrum strongly deviates from a thermal distribution. The electrons of the tenuous plasma, accelerated at the expense of the ion bulk-kinetic energy, are thermalized. Since they are forced to follow the ion spatial distribution to maintain charge neutrality, their density is neither uniform nor steady in the downstream region. We conclude that the forward shock has not yet fully developed. Although in asymmetric unmagnetized-plasma collisions kinetic instabilities generate ample magnetic turbulence in the tenuous-plasma region, the magnetic field is still too weak to efficiently decouple the dilute-ion beam from the dense slab and to force the ion-beam reflection needed to maintain the shock.
The contribution of the reflected tenuous ions to the total density of ions counterstreaming against the incoming tenuous beam and to the plasma compression at the CD continuously increases during the simulation. However, downstream of the forward shock the density of tenuous ions begins to exceed that of dense ions only at a very late stage. Reflection of the tenuous ions is most effective downstream and in the foreshock region with strong current filaments (see region with $3100 \lesssim x / \lambda_{\text {si }} \lesssim 3300$ in Figure 3 ) but also takes place in the ion precursor farther upstream where plasma cavities nonlinearly grow and expand $\left(3300 \lesssim x / \lambda_{\text {si }} \lesssim 3700\right)$. This can also be observed in Figure $2(\mathrm{~g})$. One can note that the locations at which the ions are decelerated and deflected well coincide with the density compressions caused by the expanding cavities (compare, e.g., $x / \lambda_{\mathrm{si}} \approx 3340$ and 3390 in Figures $2(\mathrm{~d}),(\mathrm{e})$, and $(\mathrm{g})$ ). As the electron phase-space plots reveal (Figures 2(f) and $3(\mathrm{~d})$ ), the ion deceleration at these locations is accompanied by localized electron heating.

As noted above, in the simulation frame the system of forward and reverse shocks separated by a CD moves toward the incoming dense-plasma beam with velocity $\beta_{\mathrm{CD}}=-0.06$. This speed defines the $C D$ frame or downstream rest frame, in which particle spectra are calculated. Particle spectra in kinetic energy, $E_{\text {kin }}=(\gamma-1) m_{l} c^{2}\left(m_{l}=m_{e}, m_{i}\right)$, are presented in Figure 4 . They are based on all particles located downstream of the forward shock in a slice of width $\sim 20 \lambda_{\text {si }}$, centered at $x / \lambda_{\mathrm{si}} \approx$ 3120. At this location, the contribution of dense electrons (not shown) to the total number density and kinetic energy density is negligible. The distribution of tenuous electrons is isotropic and quasi-thermal with no evidence of a suprathermal tail. Thus, the efficiency of any electron-injection process that may have occurred in the forward-shock region in the unmagnetized plasma is very small (compare Section 3.2.5). The electrons account for $37 \%$ of the total particle kinetic energy, dense ions contribute another $38 \%$, and dilute ions carry the remaining $25 \%$. The ion distributions are very anisotropic, which impacts the energy spectra (compare Figures 3(e) and (f)). In the spectrum of tenuous ions, the decelerated beam largely contributes to a peak around $E_{\text {kin }} \approx 0.4$; heated beam particles and particles returning upstream build the high-energy tail. The dense ions form a separate colder distribution that is not mixed in phase space with the dilute component. The high-energy tail in the spectrum is formed by particles that are reflected back to the dense-plasma region. These features again demonstrate that the forward shock is yet in the formation phase and the processes of energy exchange/equilibration between the electron and ion components are still ongoing.

\subsubsection{Reverse-shock Transition}

An important feature to be noted from Figure 3 is that the forward shock is a region in which the ions of the dense beam are strongly deflected and accelerated toward negative momenta, up to the initial momentum of the tenuous ions (see Figure 4). In consequence, these particles are able to cross the $\mathrm{CD}$ back to the reverse-shock region to form there a population of hot and energetic ions counterstreaming against the incoming dense-plasma beam. This component of the dense ions can be clearly seen in the phase-space distribution displayed in Figure 3(e). The counterstreaming dense-ion beam is generated during the entire evolution of the system, slowly building the plasma compression associated with the reverse shock left of the CD. 
By the time the simulation ends, the reverse-shock transition has not yet reached the compression ratio of a hydrodynamic shock (upper dashed line in Figure 3(a)). Although the plasma density is homogeneous in the vicinity of the $\mathrm{CD}$, particle distributions, including dense electrons, are strongly anisotropic. Upstream of the density compression, the oblique-mode instability amplifies the transverse magnetic field, resulting in structures analogous to those observed earlier. The instability is generated in the entire extended region that is populated with particles of the hot tenuous-ion beam. The magnetic field is very weak and exerts no visible modulation to the plasma density. This is largely due to temperature effects. The tenuous-ion beam is accompanied by a cloud of very hot tenuous electrons that provide charge neutrality and also cause substantial heating of the dense electrons. The oblique-filamentary magnetic structures advected and compressed in the reverse shock are further amplified through interactions with energetic dense-ion particles reflected in the $\mathrm{CD}$ region, so that a weak magnetic precursor to the reverse shock forms.

\subsection{Magnetized Plasma}

The strength of the mean magnetic field assumed for the magnetized run M1 has been chosen to characterize the physical effects introduced to the system described in Section 3.1 by the presence of the homogeneous flow-aligned field component. The physical conditions represent a weakly magnetized plasma, in which strong shock waves should form. As checked with additional test runs, systems with lower magnetic field strength show characteristics intermediate between those observed for runs $\mathrm{U} 1$ and $\mathrm{M} 1$.

\subsubsection{Early Evolution and Filamentation-like Instability in the Dense-plasma Region}

Figure 5 shows the structure of the collision region in magnetized plasma at early time, $t=437 \omega_{\mathrm{pi}}^{-1}$. As in the unmagnetized simulation (compare Figure 1), the observed filamentation of plasma and the associated perpendicular magnetic turbulence result from Weibel-like instabilities. A direct comparison of Figures 1 and 5 reveals that several characteristics of the magnetized system are analogous to those described for the unmagnetized case. In particular, the magnetic-energy density in the tenuous-plasma region exceeds the turbulent-field energy reached in the dense plasma left of the CD. The similarities are also seen in the electromagnetic and density structure in the vicinity of the $C D$ and in the tenuous-plasma region.

Notably different from the unmagnetized case is the structure of the dense-plasma region, in which an almost purely perpendicular filamentation-like mode is produced at the head of the tenuous-ion beam counterstreaming against the incoming dense plasma. This mode appears in the out-of-plane magnetic field, $B_{z}$, which is strongly amplified. The large-scale distributions of particle densities (Figures 5(d)-(g)) show that the density modulations associated with this mode first appear in the tenuous ions that form narrow filaments with large density contrast $\left(x / \lambda_{\text {si }} \lesssim 3020\right)$. Behind these structures, filamentary modulations develop in the densities of dense electrons and ions (3010 $\left.\lesssim x / \lambda_{\text {si }} \lesssim 3040\right)$. These filaments merge and grow in size, but their density contrast decreases farther downstream of the tenuous-ion flow $\left(3040 \lesssim x / \lambda_{\text {si }} \lesssim 3070\right)$, where magnetic field filaments are further amplified through mergers.

The nature of the filamentation-like mode can be verified with linear analysis. In Figure 6, we show the growth rate in reduced-wavevector space $\left(Z_{\|}, Z_{\perp}\right), Z_{i}=k_{i} v_{L} / \omega_{\text {pe }, L}$, for

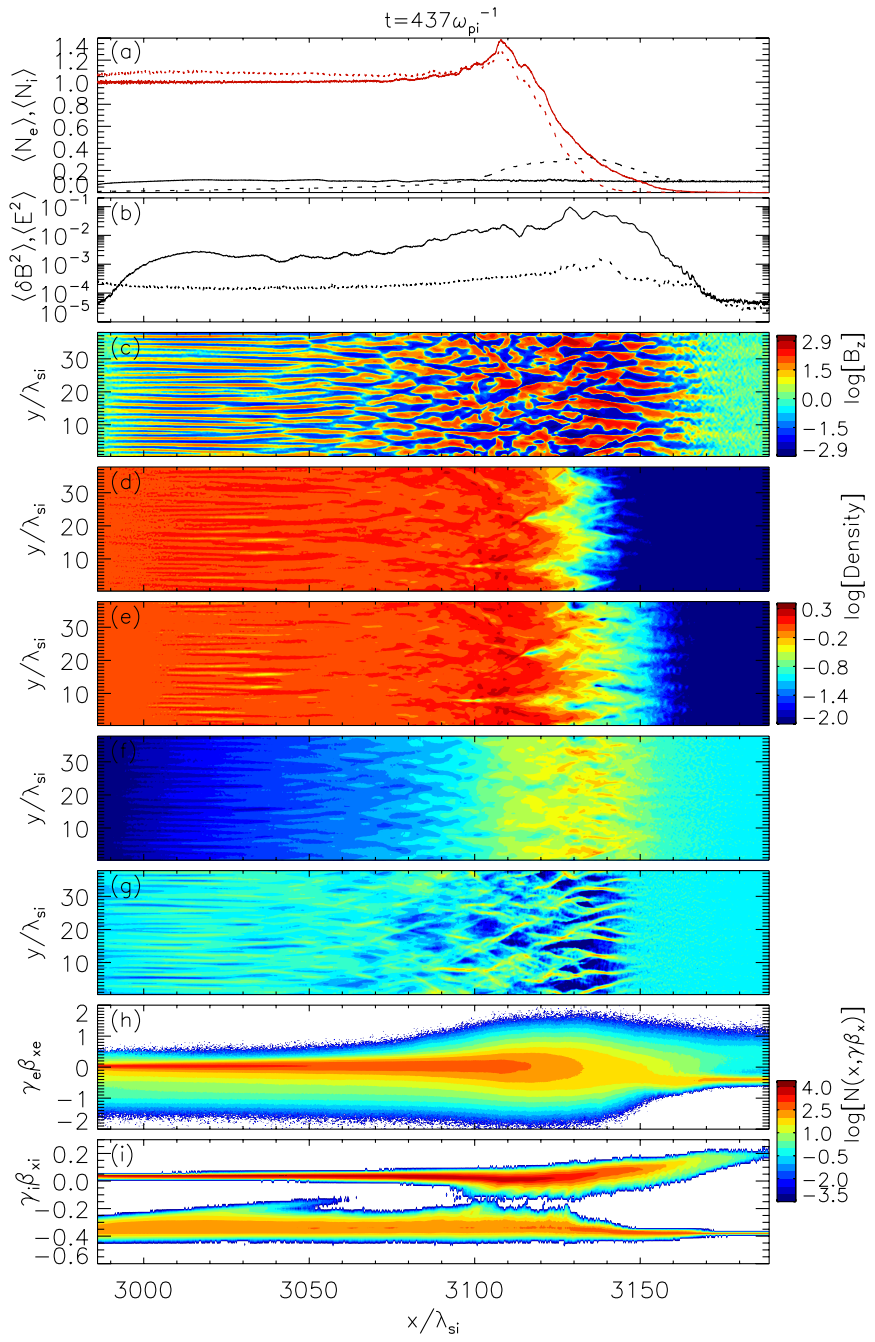

Figure 5. Structure of the collision region at time $t=437 \omega_{\mathrm{pi}}^{-1}$ for the magnetized run M1 (see Figure 1). Note that the mean magnetic field is subtracted in the magnetic-energy-density profile in panel (b). The energy density associated with the mean field component is $7.9 \times 10^{-3}$ in simulation units.

(A color version of this figure is available in the online journal.)

parameters that should represent the conditions at the head of the tenuous-ion beam. The calculations assume an initial density contrast of 0.1 between the tenuous and the dense-ion beam and the corresponding initial injection velocities. The charge and current of the dilute-ion beam are to $90 \%$ balanced by electrons of the dense beam reflected during the initial plasma collision; the remaining portion is provided by tenuous electrons leaking across the CD (see Figure 5). The reflected dense electrons are assumed to move with the bulk of the tenuous beam to account for the current balance. Although the plasma beams are heated in the simulation, we perform our calculations in the zerotemperature approximation. However, in Figure 6 we present only the low- $k$ part of the spectrum, related to instabilities that involve the ions, and we do not show the two-stream electrostatic modes at high wavenumbers that grow strongly in a cold plasma but are suppressed at finite temperature. The perpendicular wavevector of the dominant unstable mode, $Z_{\perp}=0.0138$, is in perfect agreement with $Z_{\perp}=0.0125-0.0140$ measured in the simulation $\left(\lambda \simeq 2.25-2.5 \lambda_{\text {si }}\right.$ ) and so is a nearly purely transverse character of the mode-the predicted $Z_{\perp} / Z_{\|} \approx 15$. This suggests that the wavelength of the mode is not sensitive to temperature effects, although the growth rate appears to 


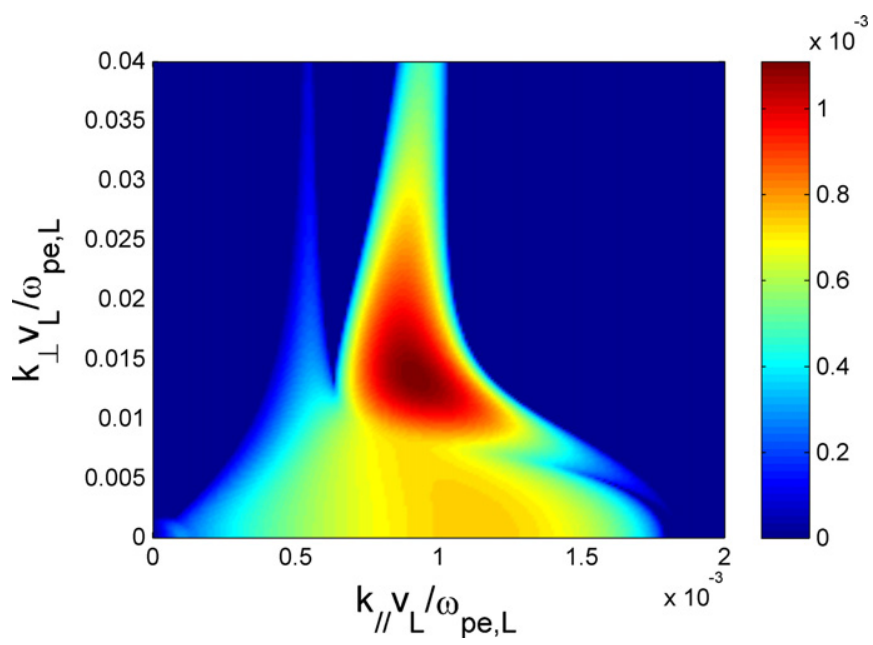

Figure 6. Linear growth rate (in units of the electron plasma frequency of the dense plasma, $\omega_{\mathrm{pe}, L}^{-1}$ ) of unstable modes as a function of the flow-aligned and perpendicular wavenumber $\left(k_{\|}\right.$and $\left.k_{\perp}\right)$, assuming parameters representing the conditions at the head of the tenuous-ion beam propagating in the dense-plasma upstream of the reverse shock (run M1). For a given $\mathbf{k}$, the growth rate of the most unstable mode is plotted, and we present only the low- $k$ part of the spectrum that does not contain two-stream electrostatic modes.

(A color version of this figure is available in the online journal.)

be reduced in warm plasma. We have further confirmed this with additional test simulations that assumed higher initial temperatures of the colliding beams (see also Section 3.2.4). Our large-scale run M2, with $m_{i} / m_{e}=20$, together with another set of test runs that incorporated larger ion-electron mass ratios of $m_{i} / m_{e}=100$ and 200, reveals that a critical parameter determining the strength of this unstable mode is ion inertia. The effect of the magnetic turbulence in the collision region on the motion of tenuous ions that pass through it is stronger for lower-mass particles and results in a warm beam for run M2 (see also Appendix A). Instead, for larger mass ratios the physical conditions in the test simulations approximate a cold-plasma limit of the linear analysis.

The presence of the filamentation-like mode significantly influences the dynamical evolution of the system. The nonlinear development of the instability in the dense-plasma region is presented in Figure 7 as a sequence of snapshots that show the $B_{z}$ magnetic field component and density distributions of dense and tenuous ions for $t \omega_{\mathrm{pi}}=437,655,874$, and 1093 . The distribution of dense electrons closely follows that of the dense ions. We also show in Figure 8 the average profiles of particle densities and electromagnetic-energy densities together with the phase-space distributions across the whole collision region for $t \omega_{\mathrm{pi}}=1093$ and 1801 . The filamentary modulations in the dense plasma that were formed behind the transverse tenuous-ion density filaments during the linear phase of the instability $\left(t=437 \omega_{\mathrm{pi}}^{-1}\right.$, Figure $\left.7(\mathrm{a})\right)$ turn in the later stage into dense-plasma cavities surrounded by regions of enhanced plasma density. These structures form only in the dense plasma and can be explained as resulting from the magnetic bubbles that are created through localized accumulation of magnetic energy caused by merging of strong current filaments (see, e.g., Murphy et al. 2010). The pressure gradient at the magnetic bubbles pushes the dense plasma out and causes the growth of the cavities. The subsequent radial expansion of the voids into each other results in further plasma compression and thus magnetic field
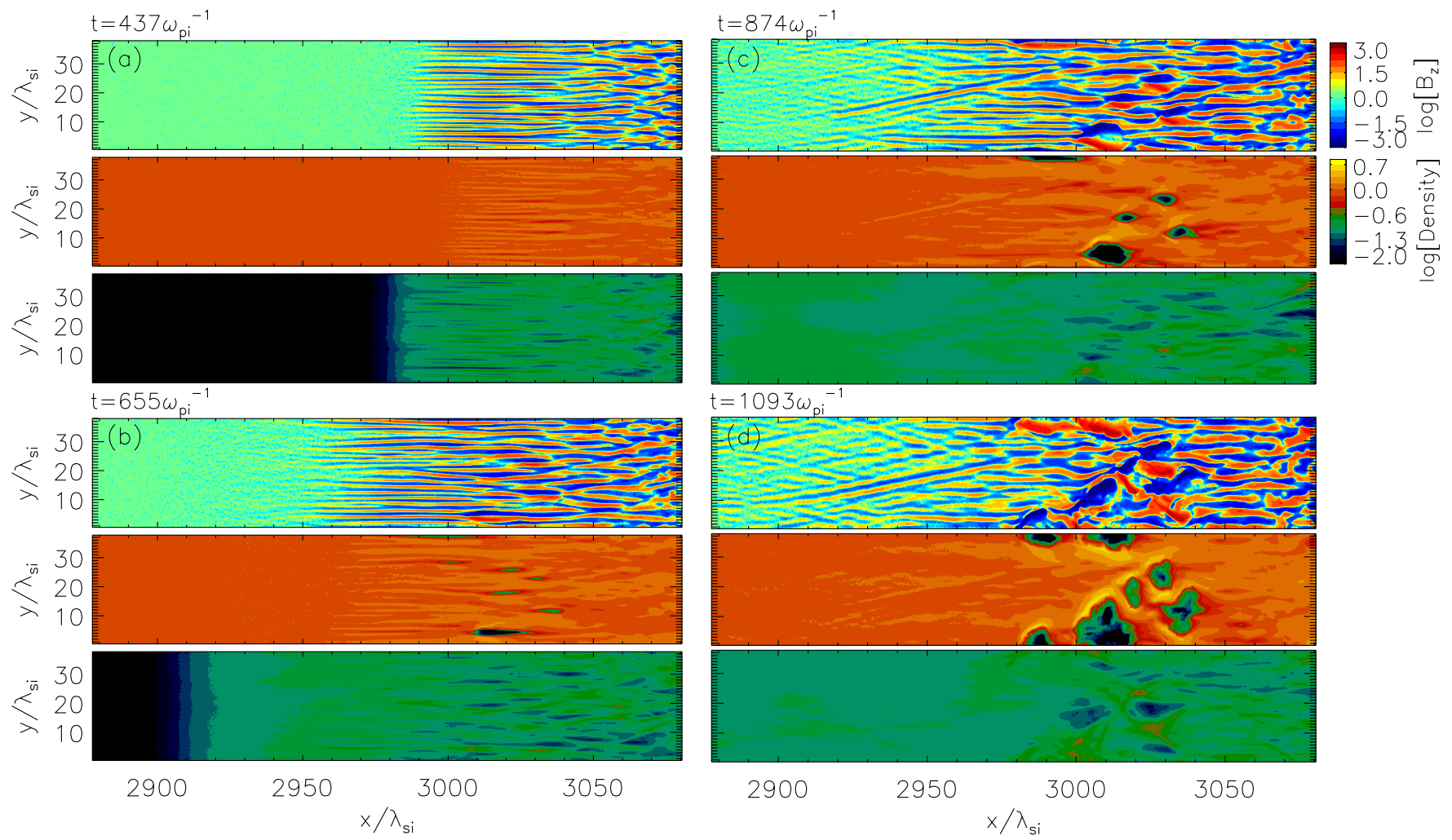

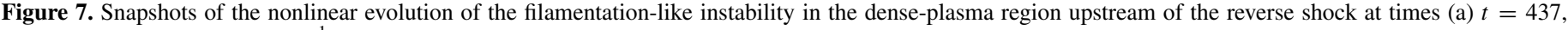

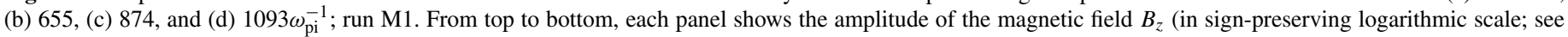
Figure 1(c)), the density of dense ions, and the density of tenuous ions, both normalized to the far-upstream density of the dense plasma.

(A color version of this figure is available in the online journal.) 

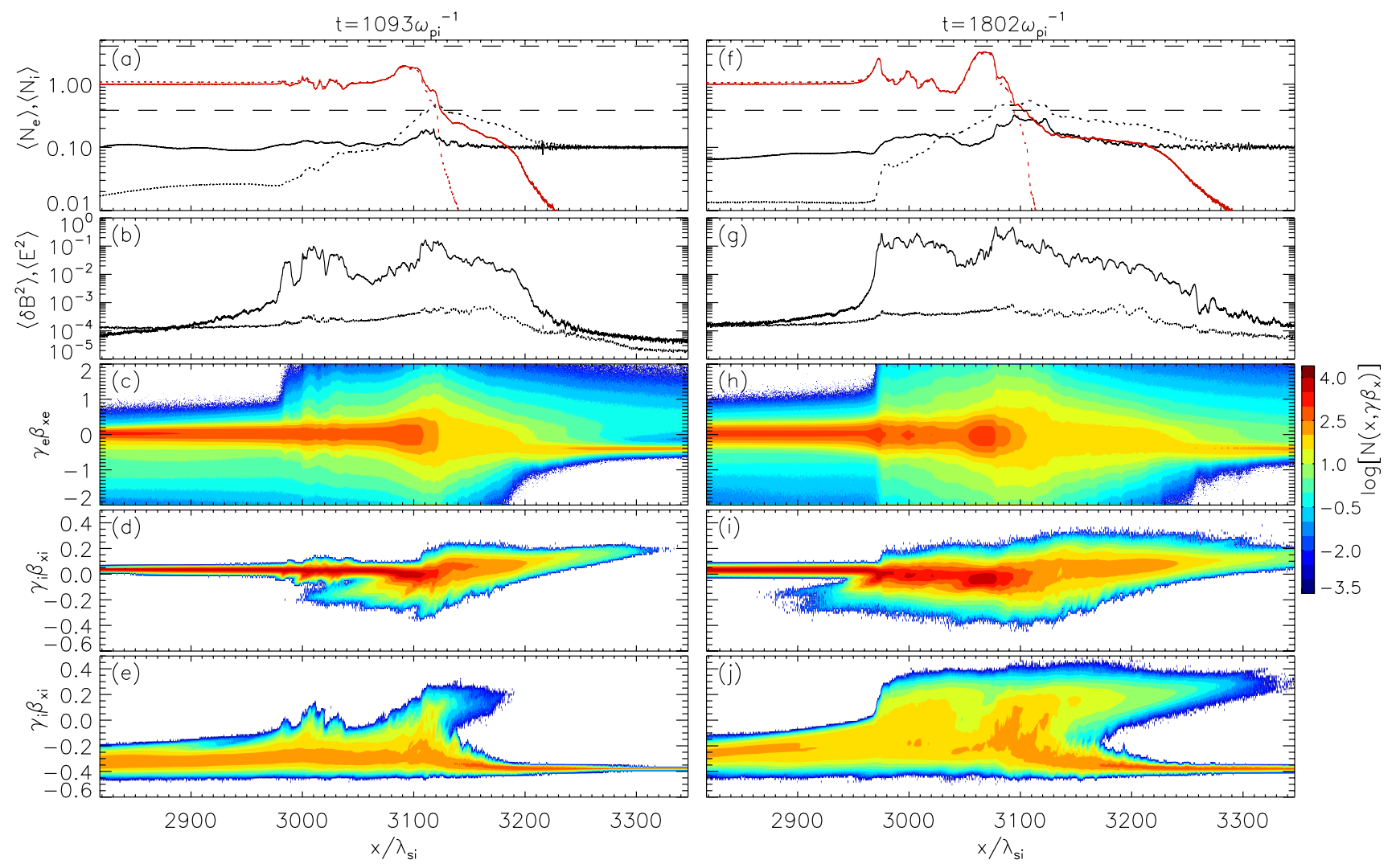

Figure 8. Structure of the collision region in the magnetized simulation M1, at time $t=1093 \omega_{\mathrm{pi}}^{-1}$ (left panel, a-e; compare Figure $\left.7(\mathrm{~d})\right)$ and $t=1801 \omega_{\mathrm{pi}}^{-1}($ right panel, $\mathrm{f}-\mathrm{j}$ ). From top to bottom each panel shows profiles of the average particle-number density, profiles of the average magnetic and electric energy density (see Figures 1 (a) and (b)), the longitudinal phase-space distribution for both electron species, and the phase space separately for the dense- and tenuous-plasma ions. In panels (a) and (f), the horizontal dashed lines mark the hydrodynamic compression level of $N_{\mathrm{fM}, \mathrm{d}} / N_{\mathrm{fM}, \mathrm{u}}=3.86$ for the forward shock (lower line) and $N_{\mathrm{rM}, \mathrm{d}} / N_{\mathrm{rM}, \mathrm{u}}=4.02$ for the reverse shock (upper line). The mean magnetic field with energy density of $7.9 \times 10^{-3}$ is subtracted in the magnetic-energy-density profiles in panels (b) and ( $\mathrm{g}$ ).

(A color version of this figure is available in the online journal.)

amplification. Similar in appearance to the dense-plasma voids, narrow filaments in the tenuous ions, which initially emerge at the front of the beam, later merge, grow in size, and eventually disperse to develop a nearly homogeneous density distribution. The growth of the filaments can be traced through the magnetic field structures in Figure 7. They demonstrate that the character of the unstable mode evolves into an oblique mode that provides only a moderate magnetic field amplification, compared to the level acquired through the initial filamentation-like mode (compare the average magnetic field profiles in Figure 5(b) for $x / \lambda_{\mathrm{si}} \lesssim 3050$ and in Figure $8(\mathrm{~b})$ for $x / \lambda_{\mathrm{si}} \lesssim 2950$ ). The tenuous ions remain unaffected by the pressure gradient at the magnetic bubbles due to their rapid motion. The density modulations in this plasma component seen around the cavities are instead the result of the nonlinear development of streaming instabilities that in this region become further enhanced by the approaching denser stream of tenuous electrons and dense ions reflected in the vicinity of the $\mathrm{CD}$. This dense-ion component can be clearly seen in Figure 8 (d) for $3000 \lesssim x / \lambda_{\text {si }} \lesssim 3100$.

The formation of voids in the dense plasma greatly affects the subsequent evolution of the system. The cavities observed for $2970 \lesssim x / \lambda_{\text {si }} \lesssim 3050$ at $t=1093 \omega_{\mathrm{pi}}^{-1}$ (Figure 7(d)) can be seen as modulations in the average density of the dense plasma, which are associated with a broad peak in the magneticenergy density (Figures 8(a) and (b)) that is comparable to the magnetic-turbulence level generated in the tenuous-plasma region to the right of the $\mathrm{CD}$. This strong field perturbs particle trajectories, thus enforcing the reflection of the interpenetrating beams into their respective upstream regions. One can observe this effect in Figures 8(d) and (e). Localized particle reflection in the region of the cavities is independent of beam deceleration and reflection processes operating in the vicinity of the $\mathrm{CD}\left(3050 \lesssim x / \lambda_{\text {si }} \lesssim 3150\right)$. Due to the large strength of the magnetic field, which is continuously amplified by the compression between the expanding voids, particle reflection is very efficient. One observes in the right panel of Figure 8 that by the time $t=1801 \omega_{\mathrm{pi}}^{-1}$ returning ions of both the dense and the tenuous plasma form almost homogeneous populations in the extended region around the $\mathrm{CD}$. The region of cavities thus constitutes a barrier for the incoming beams that causes the accumulation of particles of each plasma component on their side of this barrier. The average-density profiles (Figure 8(f)) and phase-space distributions (Figures 8(h)-(j)) clearly illustrate this behavior. Note in particular the greatly increased contribution of the tenuous ions to the total density of ions that counterstream against the incoming tenuous beam right from the $\mathrm{CD}$. Also, the dense-plasma region has become largely depleted of electrons of the tenuous component that in addition have experienced strong heating in the region of cavities.

The filamentation-like instability in the dense-plasma region thus provides a mechanism to efficiently decouple the colliding ion beams. Compared to the case of unmagnetized plasma, the presence of the mean magnetic field enables an efficient formation of the forward and reverse shocks, whose subsequent evolution is largely independent of the initial conditions. 


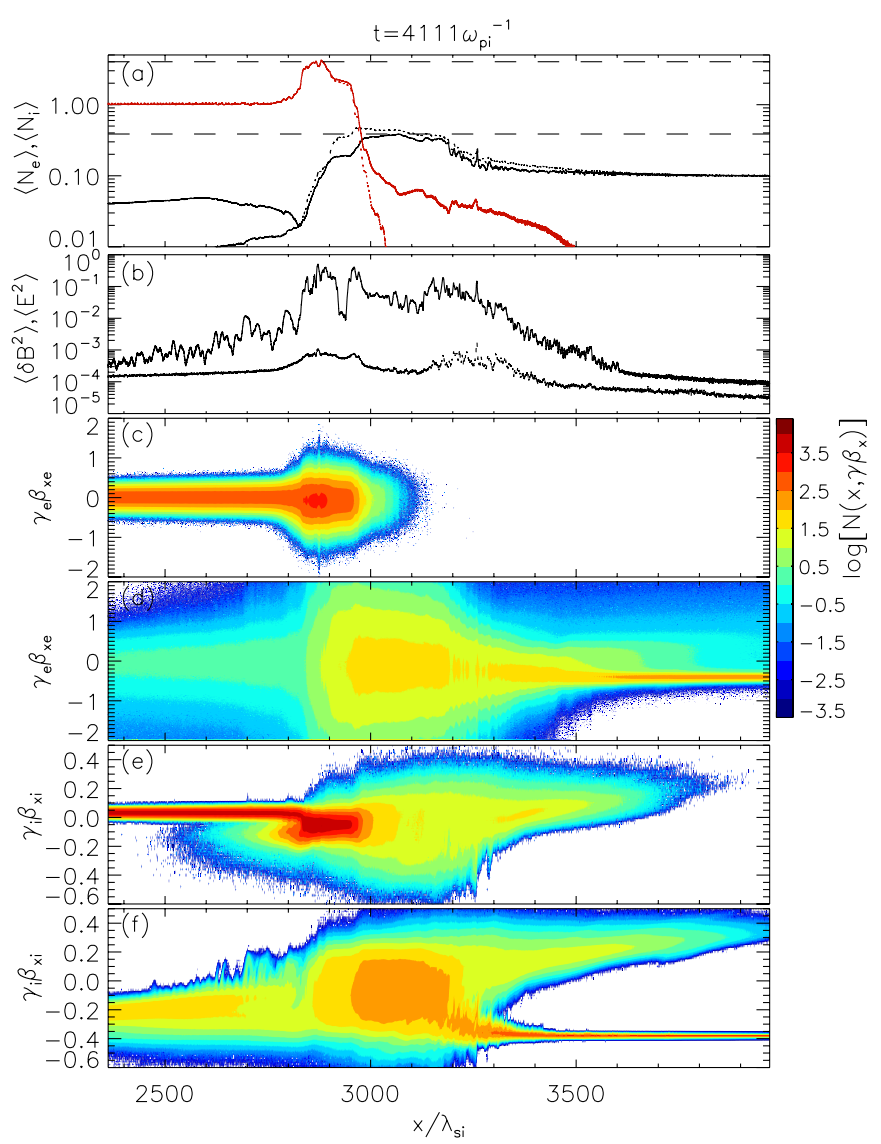

Figure 9. Structure of the collision region at the end of the simulation M1 at time $t=4111 \omega_{\mathrm{pi}}^{-1}$ (see Figure 3). The horizontal dashed lines in panel (a) mark the hydrodynamic compression level for the forward and reverse shocks (see Figure 8). The mean magnetic field with energy density of $7.9 \times 10^{-3}$ is subtracted in the magnetic-energy-density profile in panel (b).

(A color version of this figure is available in the online journal.)

\subsubsection{Late-stage Structure of the Double-shock System}

The structure of the collision region at the end of the simulation at time $t=4111 \omega_{\mathrm{pi}}^{-1}$ is presented in Figure 9 (to be compared with Figure 3). One can clearly see a system of forward and reverse shocks separated by a CD. The shocks are visible as enhancement in the number density at $x / \lambda_{\mathrm{si}} \approx 3200$ and $x / \lambda_{\mathrm{si}} \approx 2830$ for the forward and reverse shocks, respectively. The compression ratio at each shock reaches $\sim 4$, in agreement with the hydrodynamical jump conditions for nonrelativistic gas with $\Gamma_{\mathrm{M}}=5 / 3$, which predict $N_{\mathrm{fM}, \mathrm{d}} / N_{\mathrm{fM}, \mathrm{u}}=3.86$ and $N_{\mathrm{rM}, \mathrm{d}} / N_{\mathrm{rM}, \mathrm{u}}=4.02$ in the simulation frame for the forward and reverse shocks, respectively (upper and lower dashed lines in Figure 9(a)). The shock velocities measured in run M1 are also consistent with theoretical steady-state values of $\beta_{\mathrm{f}, \mathrm{M}}=0.04$ and $\beta_{\mathrm{r}, \mathrm{M}}=-0.09$ in the simulation frame. The $\mathrm{CD}$ moves with a speed close to the predicted $\beta_{\mathrm{CD}}=-0.06$ and at the end of the run is located at $x / \lambda_{\mathrm{si}} \approx 2950$. Note that the location of the CD is shifted to the left compared to that in the unmagnetized run. Only in the magnetized case do the shock-formation processes allow for an efficient heating of the tenuous plasma, whose pressure is sufficiently high to drive bulk-plasma motion already at an early stage.

As one can see in Figure 9(a), the ion compression at the forward shock is almost entirely provided by the tenuous plasma (see unmagnetized simulation U1). Although, as evidenced by the ion phase-space distributions in Figures 9(e) and (f), the contribution of the dense ions to the number density of particles returning upstream is comparable to that of the tenuous ions, the latter represent a more directed and faster flow deep in the forward-shock precursor and also completely dominate the precursor dynamics farther upstream (beyond $x / \lambda_{\text {si }} \approx 3700$ ). Thus, late in the simulation the forward shock begins to resemble a self-propagating structure that is exclusively maintained by shock-reflected particles, independent of the processes operating at and behind the CD. This is not yet the case in the denseplasma region. Although the dynamics of the deep reverseshock precursor $\left(2750 \lesssim x / \lambda_{\mathrm{si}} \lesssim 2820\right)$ is solely governed by the shock-reflected dense ions, the physics farther upstream is still determined by the interaction of counterstreaming beams of dense and tenuous ions (see below). Note, however, that the tenuous-ion beam in the reverse-shock precursor, which has diluted, slowed-down, and heated, is at this stage decoupled from the main tenuous-plasma beam coming in from the right of the CD (Figure 9(f)).

The turbulent electromagnetic field in the collision region is strongly amplified throughout the simulation to eventually reach a level about an order of magnitude larger than that achieved in unmagnetized conditions. One can see in Figure 9(b) that the turbulence is almost purely magnetic, and both the forward and the reverse shocks develop strong magnetic precursors. The energy density of the magnetic field in the forward-shock region peaks at the shock front and then decays downstream (Kato \& Takabe 2008). This turbulence is mostly generated when the forward shock is self-propagating. The second maximum to the immediate right of the $\mathrm{CD}$ arises from the amplified magnetic turbulence produced via filamentation instabilities during the early evolution of the system, before the ion beams have become decoupled from each other. The broad peak in the magnetic field energy density to the left of the CD has largely evolved from the magnetic turbulence generated earlier in the region of cavities (Figure 8). It also constitutes the shock-compressed magnetic turbulence produced upstream of the reverse shock after the cavities in the dense plasma have dissipated to form the downstream region of the reverse shock.

\subsubsection{Structure of the Forward-shock Transition}

Figure 10 presents the structure of the forward-shock transition at time $t=3499 \omega_{\mathrm{pi}}^{-1}$. This time was chosen to display the magnetic field amplitude $B_{z}$ (Figure 10(a)) and the density of tenuous ions (Figure 10(b)) with correct aspect ratio; the characteristics discussed are the same as those observed at $t=4111 \omega_{\mathrm{pi}}^{-1}$ (Figure 9). The tenuous-electron distribution (not shown) closely follows that of tenuous ions; they also provide charge balance to the low-density dense ions that occupy the volume between tenuous-ion filaments. As seen in Figure 10, the main source of turbulent magnetic field is the electromagnetic Weibel-type instability. Strong nonlinear effects of the interaction of returning particle beams with electrostatically induced parallel density structures, reported in the forward-shock precursor for the unmagnetized case, are not observed. The two-stream instability between incoming and returning electron beams generates electrostatic perturbations far upstream that lead to nonlinear charge-density waves in the precursor (for $x / \lambda_{\text {si }} \gtrsim 3450$ in Figure $10(\mathrm{~b})$ ), but the interaction of the density modulations with the reflected ions does not result in the formation of plasma cavities. The growth and expansion of parallel density fluctuations are suppressed by the mean magnetic field.

The tenuous-plasma filaments, which pinch and merge in the forward-shock foot, break up at the shock interface and 


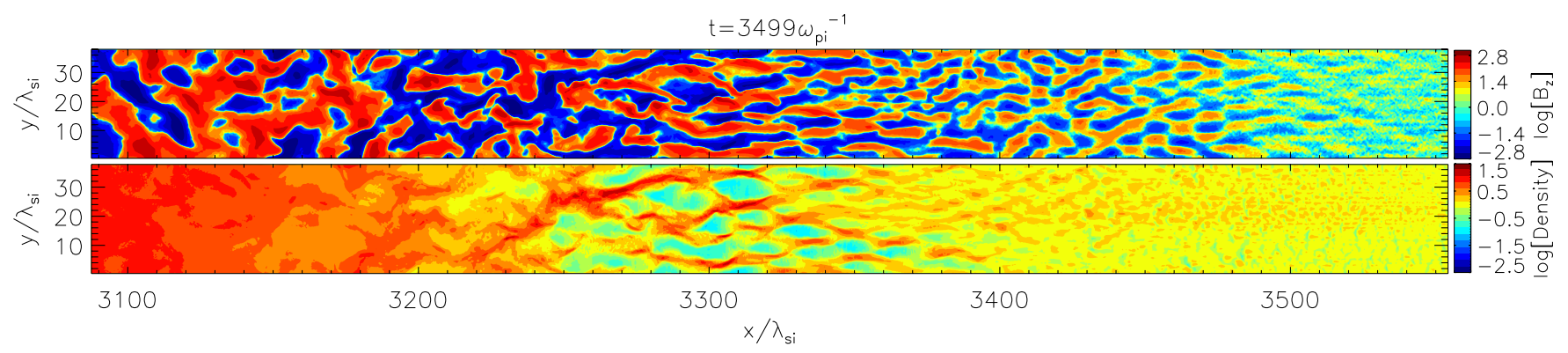

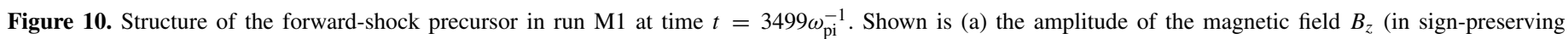
logarithmic scale; see Figure 1(c)) and (b) the density of tenuous ions normalized to the far-upstream density of the dilute plasma.

(A color version of this figure is available in the online journal.)

dissipate to form a nearly homogeneous density distribution downstream of the shock $\left(x / \lambda_{\text {si }} \lesssim 3150\right.$ in Figure $\left.10(b)\right)$. The disintegration of the tenuous-ion beam downstream of the forward shock was not observed in the unmagnetized simulation U1. Nevertheless, although the downstream tenuous-ion density is close to uniform, they are not fully thermalized (see below), which indicates that the forward-shock evolution has not fully reached a steady state by the end of the simulation.

\subsubsection{Structure of the Reverse-shock Transition}

As discussed in Section 3.2.1, strong fluctuations in the density of the dense plasma and the magnetic field resulting from a filamentation-like instability in the region of cavities began to form an obstacle for the incoming tenuous plasma and eventually caused a depletion of these particles in the dense-beam region upstream of the dense-plasma voids. In addition, the tenuous ions at the head of the dilute-ion beam were heated and the filaments dispersed. In effect, the character of the unstable magnetic mode has changed from almost purely perpendicular to slowly growing oblique turbulence, with characteristics similar to those described for the reverseshock precursor in the unmagnetized run U1 (Section 3.1.3). As in the unmagnetized case, the oblique magnetic mode is generated wherever the tenuous-ion beam propagates in the upstream region of the reverse shock. However, the presence of the mean magnetic field triggers an additional plasma mode in the system that slowly grows and eventually dominates the magnetic-turbulence spectrum in the reverse-shock precursor.

Figure 11 presents the reverse-shock transition at time $t=$ $3061 \omega_{\mathrm{pi}}^{-1}$, at which the initial oblique mode has already attenuated. As seen in Figures 11(b) and (c), which show the $B_{y}$ and $B_{z}$ magnetic field components, respectively, the unstable mode represents an almost purely parallel magnetic wave. The wave is left-hand circularly polarized ${ }^{9}$ and in the rest frame of the denseplasma beam moves antiparallel to the mean magnetic field with phase velocity $v_{\mathrm{ph}} \approx 7.9 \times 10^{-3} c \simeq 1.4 v_{\mathrm{A}, \mathrm{L}}$. Therefore, in the frame of the reverse shock the wave is carried toward the shock with the plasma flow. The wavelength of the mode is $\lambda \sim 15 \lambda_{\text {si }}$, and its frequency is $\omega \simeq 0.58 \Omega_{i}$. These characteristics do not significantly vary over the precursor region.

To understand the nature of the observed parallel mode, a linear analysis has been performed. It is analogous to the analysis for the filamentation-like mode in Section 3.2.1 but uses a modified set of parameters because the physical conditions in the reverse-shock precursor have changed. The calculations

\footnotetext{
9 For $\mathbf{B}_{0}=B_{0, \mathrm{x}} \hat{\mathbf{x}}$ and $\mathbf{k}=k_{x} \hat{\mathbf{x}}$, a plane electromagnetic wave of the form $\mathbf{B}_{1} \exp i(\mathbf{k} \cdot \mathbf{x}-\omega t), \mathbf{B}_{1}=B_{1, \mathrm{y}} \hat{\mathbf{y}}+B_{1, \mathrm{z}} \hat{\mathbf{z}}$ is left-hand circularly polarized if $B_{1, \mathrm{z}}=-i B_{1, \mathrm{y}}$.
}

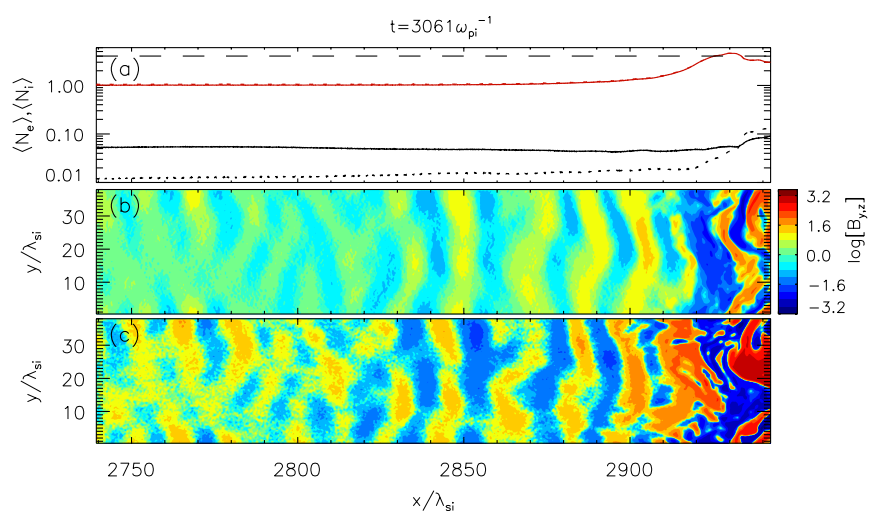

Figure 11. Structure of the reverse-shock transition in run $M 1$ at time $t=3061 \omega_{\mathrm{pi}}^{-1}$. Shown are (a) profiles of the average particle-number density (see Figure 1(a)) and the amplitude of magnetic field components, $B_{y}$ (b) and $B_{z}$ (c). The horizontal dashed line in panel (a) marks the hydrodynamic compression level of $N_{\mathrm{rM}, \mathrm{d}} / N_{\mathrm{rM}, \mathrm{u}}=4.02$ for the reverse shock. Note that the field components are in sign-preserving logarithmic units, e.g., for $B_{z}$ as $\operatorname{sgn}\left(B_{z}\right)\left(3+\log \left[\max \left(10^{-3},\left|B_{z}\right|\right)\right]\right)$.

(A color version of this figure is available in the online journal.)

thus assume a density contrast of 0.05 between the dilute and the dense-ion beam (compare the average-density profiles in Figure 11(a)) and a tenuous-ion beam velocity of $v=-0.3 c$, slightly slower than the initial injection velocity $v_{\mathrm{R}}$. The charge and current of the tenuous ions are compensated mainly by returning dense electrons; electrons of the tenuous plasma represent only $5 \%$ of all returning electrons. We assume the coldplasma approximation. The results are displayed in Figure 12, in which the two-stream electrostatic modes at high wavenumbers are again not shown. The area of strongest growth at $Z_{\|} \approx$ 0.0019 and $Z_{\perp} \approx 0.012$ pertains to an oblique mode, analogous to the nearly transverse mode discussed in Section 3.2.1. The second maximum at $Z_{\perp} \approx 0$ and $Z_{\|} \approx 0.002$, or $\lambda_{\|} \approx 16 \lambda_{\text {si }}$, can be identified with our parallel mode. Note that the growth rates of both the oblique and the parallel modes are comparable to within $6 \%$ in the present setup, which was not the case for the analysis of the early-stage turbulence modes in the denseplasma region, in which a parallel mode can also be observed at $Z_{\|} \approx 0.0012$ but with a growth rate $70 \%$ of that of the dominant unstable filamentation-like mode (Figure 6).

The nature of the parallel mode can be explained by analysis of the dielectric tensor $\mathbf{T}(\mathbf{k}, \omega)$ for our system. For flow-aligned perturbations with $k_{y}=0$, the tensor $\mathbf{T}$ takes the form

$$
\mathbf{T}=\left(\begin{array}{ccc}
b & 0 & -c \\
0 & a & 0 \\
c & 0 & b
\end{array}\right),
$$




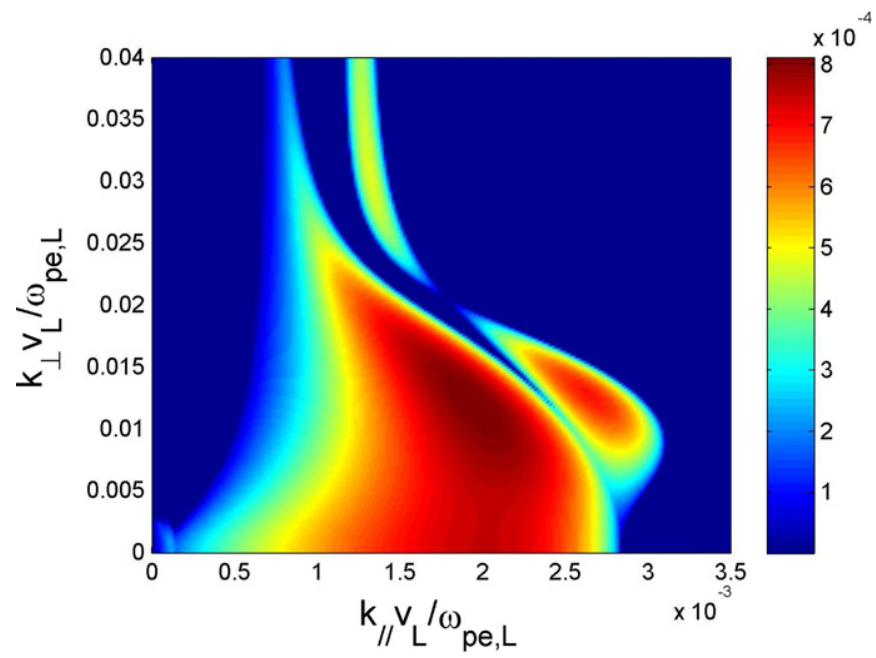

Figure 12. Linear growth rate of unstable wave modes for conditions representing the late-stage structure of the reverse-shock precursor in run M1 (see Figure 6).

(A color version of this figure is available in the online journal.)

where the tensor elements $a, b, c$ are reported in Appendix B. As one can see, the dispersion equation displays three branches, as $\operatorname{det} \mathbf{T}(\mathbf{k}, \omega)=0$ readily gives

$$
a(b+i c)(b-i c)=0 .
$$

The equation $a=0$ defines two-stream-like electronic modes at large wavevectors. As already noted, at the timescale of the present simulations, these modes quickly saturate for warm electron beams. On the ion timescale, the large-wavelength modes governing the system arise from the branches $(b+i c)=0$ and $(b-i c)=0$. When analyzing a simpler setup formed by a dilute-ion beam passing through an electron-ion plasma, the so-called non-resonant (Bell) mode, as well as the resonant one, comes from $(b-i c)=0$ (Bell 2004; Reville 2006; Winske \& Leroy 1984). In the present case, the growth-rate profile observed along the parallel axis in Figure 12 (and also in Figure 6) can equally be traced back to the same $(b-i c)=0$ branch. We can therefore identify our parallel mode with the "Bell-like" mode. However, the linear analysis predicts a frequency $\omega=0.0012 \omega_{\mathrm{pe}, L}=0.0085 \omega_{\mathrm{pi}, L} \simeq 1.5 \Omega_{i}$ that is a factor of 2.6 higher than observed in our simulation. This indicates that either the cold-plasma approach is invalid or the simplified conditions assumed for the linear analysis do not properly represent the conditions in the simulations.

To evaluate how well the simplified conditions correspond to the state of the plasma system in the simulated reverse-shock precursor and to estimate the effects of a finite temperature, two additional test runs have been performed. Both assume the conditions used in the linear analysis and periodic boundary conditions. In the first run all plasma components are cold. We refer to this simulation as to the cold test run. In the second test simulation, the plasma beams initially have temperatures as measured in the precursor. This run is referred to as the warm test run. We found that both test runs well reproduce the characteristics of the initial oblique mode, which is the fastest-growing mode in the linear analysis. The nearly homogeneous density distributions and the absence of strong density filaments, which are observed in the main simulation, are both well replicated in the warm test run. In the latter and in the magnetized run M1, the oblique mode is observed mainly through filamentation in the $B_{z}$ field component. The parallel mode appears at a later stage in both test runs. Whereas in the warm run it is the only magnetic mode, in the cold test run the oblique-mode nonlinear filaments in $B_{z}$ are clearly imprinted onto the parallel magnetic field fluctuations. These characteristics are due to thermal effects that lead to damping of the wave modes with perpendicular components because the plasma pressure opposes pinching of the filaments. Consequently, the oblique mode eventually dissipates in the warm plasma, and the parallel mode, whose linear growth rate is only slightly lower than that of the oblique mode, dominates the nonlinear evolution.

The parallel mode observed in the test runs has a wavelength $\lambda_{\|} \sim 16 \lambda_{\text {si }}\left(Z_{\|} \simeq 0.002\right)$, is left-hand circularly polarized, and propagates antiparallel to the mean magnetic field. The measured growth rate is about half the analytically derived growth rate, which is consistent with the influence of thermal effects. These results very well reproduce the properties of the parallel mode observed in the magnetized simulation. The measured frequency of the mode is $\omega \approx 1.1 \Omega_{i}$ in the cold test run and $\omega \approx 0.7 \Omega_{i}$ in the warm test run. The results of the warm test run are therefore consistent with our main simulation, which indicates that the simplified conditions well represent the real conditions in the simulations, and thus our identification of the parallel mode with the Bell-like mode is correct. The differences between the measured mode frequency and that predicted in the linear analysis likely result from thermal effects, which in the nonlinear stage modify the physical conditions, possibly introducing factors not accounted for in the linear calculations.

As one can note in Figure 11, the amplitude of the parallel magnetic field fluctuations considerably increases in the reverseshock foot. The latter is the precursor region in which a highdensity population of shock-reflected dense ions is present at $x \gtrsim 2880 \lambda_{\mathrm{si}}$. The amplification and compression of the parallel fluctuations thus provide a formation mechanism for the reverse-shock transition that is consistent with the hydrodynamic picture. Note that although the parallel magnetic mode excited in the precursor region results from the initial conditions, similar unstable modes should appear later on account of shockreflected dense plasma. In fact, our linear calculations show that the appearance of the Bell-like mode critically depends on the presence of returning dense electrons in the shock-precursor plasma, and not on the tenuous-ion beam. Therefore, one may expect that the further evolution of the reverse-shock system will be similar.

\subsubsection{Particle Distributions and Electron Injection at the Forward Shock}

As in the case of unmagnetized plasma (run U1), particle distributions are calculated in the rest frame of the downstream plasma, defined by the $\mathrm{CD}$ speed $\beta_{\mathrm{CD}}=-0.06$ in the simulation frame. Although in run M1 the downstream plasma is turbulent on large scales in the CD rest frame, these turbulent motions are slow and do not significantly distort the spectra.

For run M1 we define the forward-shock downstream region as the area of roughly constant plasma compression with ratio $\sim 4$ that is dominated by particles of the tenuous plasma whose bulk velocity is similar to that of the CD. At $t=4111 \omega_{\mathrm{pi}}^{-1}$, the downstream region spans the range $2980 \lesssim x / \lambda_{\text {si }} \lesssim 3080$ (see Figure 9). Left of this location is the CD region in which both the dense and the tenuous plasma reside and where additional processes may operate that alter the particle spectra. As for run $\mathrm{U} 1$, to calculate particle distributions we choose a slice of width $\sim 20 \lambda_{\mathrm{si}}$, now centered at $x / \lambda_{\mathrm{si}} \approx 3000$. The slice is stationary 


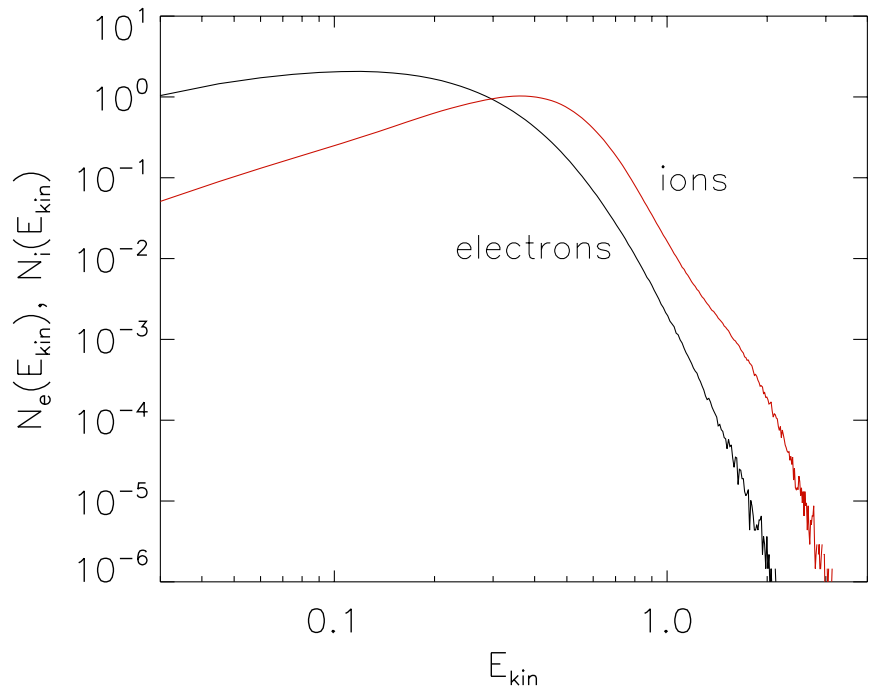

Figure 13. Kinetic-energy spectra of tenuous electrons (black line) and tenuous ions (red line) in the forward-shock downstream region at $x / \lambda_{\mathrm{si}} \approx 3000$ and time $t=4111 \omega_{\mathrm{pi}}^{-1}$ for the magnetized run M1, in the CD rest frame. The spectra are normalized and expressed in simulation units, in which $m_{e} c^{2}=0.25$.

(A color version of this figure is available in the online journal.)

with respect to the $\mathrm{CD}$, and thus the shock front moves steadily away from the location at which spectra are measured.

Figure 13 presents spectra of tenuous electrons and ions at time $t=4111 \omega_{\mathrm{pi}}^{-1}$. Only the distributions of electrons appear approximately thermalized. Visible also in Figure 9, the distribution function of tenuous ions exhibits a plateau when integrated over any two momentum coordinates, e.g., $f\left(p_{x}\right)=\int d p_{y} d p_{z} f(\boldsymbol{p})$, and consequently the kinetic-energy spectrum strongly deviates from a thermal distribution. Isotropy is approximately maintained in the plateau section of the distribution, but not in the wings where the distribution function falls off. The form of the distribution suggests that the ions have reached stability against parallel electrostatic and electromagnetic instabilities. As in the case of the unmagnetized system, in run $\mathrm{M} 1$ the total energy content in electrons is roughly the same as that in ions, in fact $2 / 3$ of it.

Figure 14 details the time evolution of the downstream electron spectrum. At all times for which spectra are presented $\left(t \omega_{\mathrm{pi}}=2406,2974,3542\right.$, and 4111, shown with black, blue, green, and red line, respectively), the forward-shock compression is well established in the extended region to the right of the $\mathrm{CD}$, and the electrons are isotropic in the downstream frame (see run U1). These spectra display a slow increase in average energy, possibly because the region, in which the distribution is calculated, is located closer to the shock front at earlier times. All spectra are slightly more peaked than a relativistic Maxwellian, which we plot for comparison. It is interesting to note that renormalizing the spectra to dimensionless energy $x=E / E_{\mathrm{kin}, \mathrm{av}}, E_{\mathrm{kin}, \mathrm{av}}$ being the average particle energy, indicates that the shape of the spectra evolves very weakly, and it is indeed mainly a continuous increase in $E_{\text {kin,av }}$ that is responsible for the spectral evolution. There is no significant indication of a suprathermal tail in the electron spectrum.

Dense-plasma particles downstream of the reverse shock are sampled in a slice of width $\sim 40 \lambda_{\text {si }}$, centered at $x / \lambda_{\text {si }} \approx 2870$, and their energy distributions in the CD rest frame at the end of run M1 are presented in Figure 15. The hydrodynamic plasma compression is fully established in this narrow region at the very

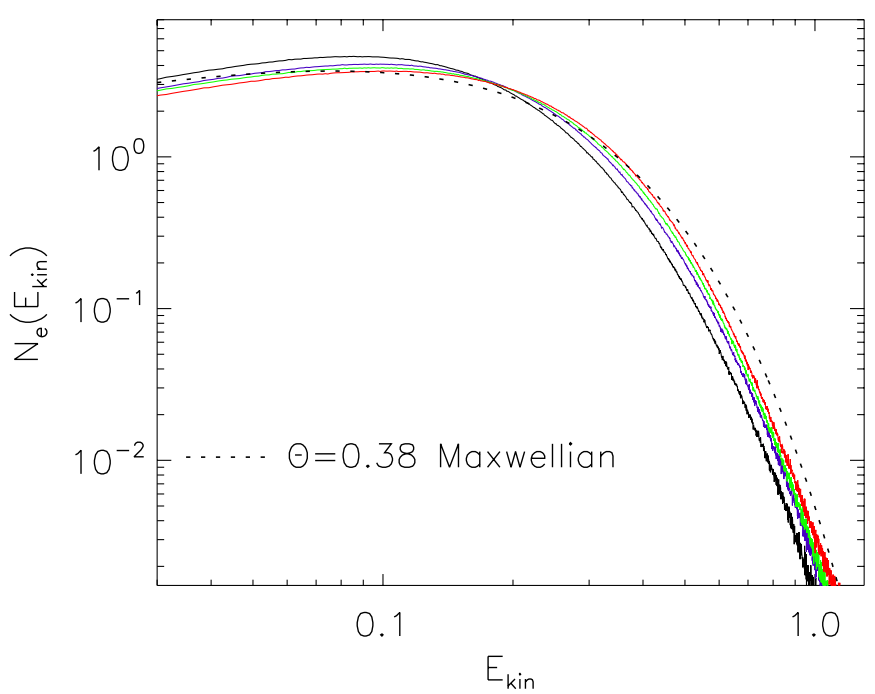

Figure 14. Tenuous-electron kinetic-energy spectra in the forward-shock downstream region at $\sim 50 \lambda_{\text {si }}$ from the CD at times $t=2406$ (black line), 2974 (blue line), 3542 (green line), and $4111 \omega_{\mathrm{pi}}^{-1}$ (red line) for run M1. All spectra are normalized and expressed in simulation units, in which $m_{e} c^{2}=0.25$. The dotted line shows a relativistic Maxwellian for comparison.

(A color version of this figure is available in the online journal.)

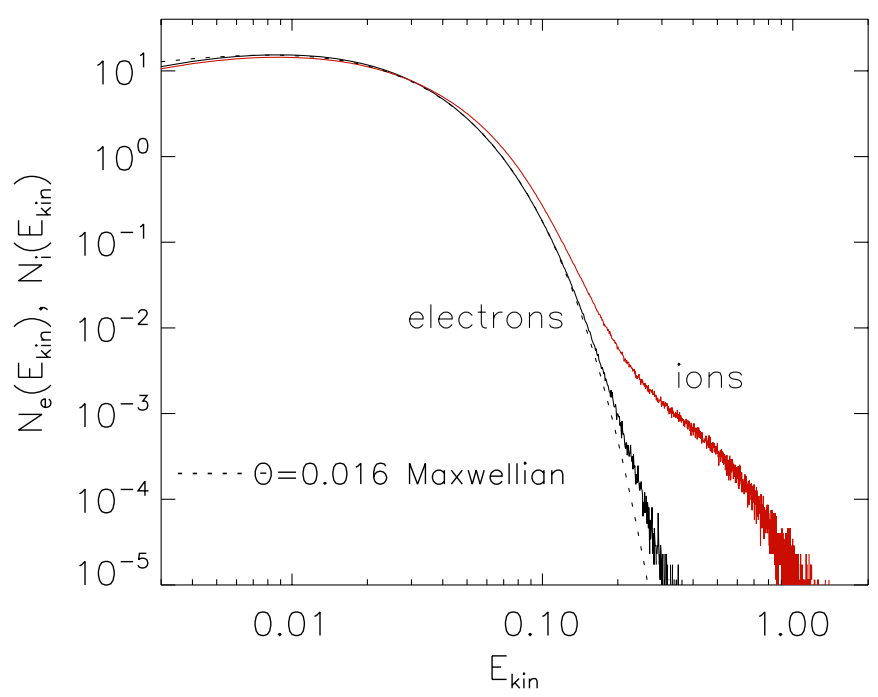

Figure 15. Kinetic-energy spectra of dense electrons (black line) and dense ions (red line) in the reverse-shock downstream region at $x / \lambda_{\mathrm{si}} \approx 2850-2890$ and time $t=4111 \omega_{\mathrm{pi}}^{-1}$ for the magnetized run M1, in the CD rest frame. The spectra are normalized and expressed in simulation units, in which $m_{e} c^{2}=0.25$. The dotted line shows a Maxwellian fit to the electron spectrum.

(A color version of this figure is available in the online journal.)

end of the simulation. The electrons and ions carry to within $10 \%$ the same kinetic energy. Only in the high-energy tail do we observe a modest anisotropy in the electron distribution. A Maxwellian fits the electron spectrum reasonably well; a slight discrepancy at high energies coincides with anisotropy. The ions are anisotropic as well; a substantial population of returning particles exists that builds a superficial high-energy tail (see Figure 9), which is therefore not indicative of ion preacceleration.

\section{SUMMARY AND DISCUSSION}

We have performed 2D3V PIC simulations of nonrelativistic plasma collisions with absent or parallel large-scale magnetic 
field. Contrary to earlier expectations, simulations by Kato \& Takabe (2008) suggested that Weibel-type instabilities can trigger shock formation in an unmagnetized environment even for collisions with nonrelativistic velocity, but fast enough to prohibit an electrostatic shock (Forslund \& Shonk 1970; Forslund \& Freidberg 1971). A pre-existing magnetic field may therefore not be necessary for the formation of nonrelativistic shocks. The question we addressed in this study is whether or not the shock structure and the particle spectra reach a steady state, and to what degree a parallel magnetic field renders shock formation more efficient. A secondary goal was to investigate the efficacy of particle pre-acceleration, which appears needed for their injection into a diffusive shock-acceleration process.

To address these questions, we follow the evolution of the system longer than was done by Kato \& Takabe (2008). Also, we are interested in asymmetric flows, i.e., the collision of plasma slabs of different density, leading to two different shocks and a $\mathrm{CD}$ that is self-consistently modeled. Emphasis has been placed on setting up the simulations as clean as possible.

The simulation parameters are chosen to be close to those of young SNRs, except that we use a reduced ion-to-electron mass ratio, $m_{i} / m_{e}=50$. About 50 years after the explosion of a Type Ia supernova, the density ratio between ejecta and ambient medium is about 10 as assumed here, if the density of the ambient gas is about 1 atom $\mathrm{cm}^{-3}$. The shock velocities are about $0.05 c$, a factor of a few lower than in our simulations. In the magnetized case, the Alfvénic Mach number of the forward shock is $M_{\mathrm{Af}}=c \beta_{\mathrm{fR}, \mathrm{M}} / v_{\mathrm{A}, \mathrm{R}} \simeq 21.1$ and that of the reverse shock is $M_{\mathrm{Ar}}=c \beta_{\mathrm{rL}, \mathrm{M}} / v_{\mathrm{A}, \mathrm{L}} \simeq 22.5$. Here, as forward shock we denote the shock propagating into the low-density (ambient) plasma; vice versa, the reverse shock travels through the dense plasma that represents the ejecta.

Our results can be summarized as follows:

1. In both the unmagnetized and magnetized cases, a doubleshock structure builds on timescales of about a thousand ion plasma times, $\omega_{\mathrm{pi}}^{-1}$. The colliding plasmas are initially very cold, so that the sonic Mach number in our simulations is high enough to prevent an electrostatic shock. We show that while nonrelativistic shocks in both unmagnetized and magnetized plasmas can be mediated by Weibel-type instabilities, the efficiency of shock-formation processes is higher when a large-scale magnetic field is present. The amplitude of magnetic turbulence generated in the collision region is an order of magnitude larger in the magnetized plasma than in unmagnetized conditions. The typical magnetization of the ambient medium, into which young SNR shocks propagate, given by $\sigma=M_{\mathrm{A}}^{-2}$, is in the range $10^{-6}<\sigma<10^{-3}$. Our magnetized run with $\sigma \approx 2 \times 10^{-3}$ thus probes an upper limit of the sigma parameter, and any realistic formation scenario of parallel shocks in SNRs should show characteristics intermediate between the two cases studied here.

2. In the unmagnetized case, small-scale electrostatic and filamentation-like instabilities operate in parallel and heat the electrons. Eventually, strong fluctuations arise in the density of electrons and ions that lead to the formation of the double-shock structure. The electron dynamics play an important role in the development of the system. Ion-ion or ion-electron streaming generally drives the turbulence, which is mainly magnetic. The exact type of an instability, however, depends on the location in the shock precursor and is generally different for forward and reverse shocks. Nonlinearities and the change of conditions during plasma advection toward the shocks render an identification with linear modes difficult. In the magnetized case, filamentation in the dense-plasma region is initially more pronounced, which we verify with linear analysis, but it quickly evolves into an oblique mode and nonlinear density fluctuations. Some of the streaming instabilities that lead to the formation of the reverse shock are similar to non-resonant modes that have been discussed in the context of cosmic-rayinduced magnetic field amplification (e.g., Bell 2004; Niemiec et al. 2008, 2010; Riquelme \& Spitkovsky 2009; Stroman et al. 2009, 2012). In both the magnetized and unmagnetized systems, magnetic field generation processes lead to stronger magnetic fields downstream of the forward shocks, rather than reverse shocks. We would thus expect synchrotron emission to originate from the magnetized structure of the forward-shock transition, which is in agreement with SNR observations.

3. The electron distributions downstream of the forward and reverse shocks are generally isotropic, whereas that is not always the case for the ions. The observed spectra suggest that the electrons have reached a statistical equilibrium and generally carry nearly as much kinetic energy as do the ions, although the latter have not yet reached an equilibrium.

4. We do not see any significant evidence of pre-acceleration either in the electron population or in the ion distribution. Note that the shock-surfing acceleration, which reportedly provides fast electron acceleration (Amano \& Hoshino 2009), should not operate in situations with absent or strictly parallel magnetic field, because $\mathbf{v} \times \mathbf{B}=0$. In our magnetized simulation, the Alfvénic Mach number is slightly below the threshold (at $M_{\mathrm{A}} \gtrsim 25$ ) for the electron-acceleration process described by Amano \& Hoshino (2010). Dieckmann et al. (2010) and Murphy et al. (2010) report efficient electron acceleration to Lorentz factors of order 100 in PIC simulations of slightly faster plasma collisions than discussed here $(0.5 c$ and $0.9 c$ instead of $\sim 0.4 c)$. In both cases the strong $\left(\Omega_{e} / \omega_{\mathrm{pe}} \sim 1\right)$ large-scale magnetic field is assumed to be obliquely oriented relative to the flow axis, which provides for more effective particle reflection in the perpendicular component of the magnetic field at the shock transitions. Being interested in ion dynamics, Gargaté \& Spitkovsky (2012) present hybrid simulations and a PIC simulation of a parallel shock with $M_{\mathrm{A}}=4.7$, about 4.5 times the magnetization assumed here. They find emergent ion acceleration very late in the PIC simulation (at $t \simeq 87 \Omega_{i}^{-1}$ ) and also during the subsequent evolution captured with their hybrid simulations. They also find that ion acceleration proceeds to higher energies if the Alfvénic Mach number is larger, although the injection efficiency decreases. In ion plasma times, our magnetized PIC simulation has similar duration as that of Gargaté \& Spitkovsky (2012) but is nearly four times shorter in ion gyro-times on account of the lower plasma magnetization assumed in our simulations. However, the low Alfvénic Mach number used in the PIC simulation in Gargaté \& Spitkovsky (2012) suppresses Weibel-type instabilities. They are also not modeled with their hybrid simulations for faster shocks (their $M_{\mathrm{A}}=31$ run should be comparable with ours). Because we demonstrate that electron dynamics are important for the evolution of the system, a direct comparison with these results is not possible. Nevertheless, all spectral structures we find coincide with anisotropic distribution functions, suggesting 
an imperfect ion-beam relaxation rather than acceleration per se.

5. We have also studied the effect of the assumed ion-toelectron mass ratio on our results. We find that although the main characteristics of the long-time evolution of the systems do not critically depend on the mass ratio within the range studied, the detailed mechanisms of reaching a steady state might be modified or even different if the assumed mass ratio is too low. The timescales for particle-energy equilibration and the efficiency of particle pre-acceleration processes might be overestimated in simulations that use a reduced ion-to-electron mass ratio.

Computer resource limitations prohibit us from studying the properties of the shock formation for close-to-realistic ion-toelectron mass ratios. Studies of the saturation of the linear phase of purely transverse ion-driven filamentation instability indicate a very low efficiency of the conversion of the ion-beam energy into magnetic field energy for a realistic mass ratio (e.g., Ren et al. 2007; Wiersma \& Achterberg 2004), suggesting that other instabilities must mediate the shock formation in astrophysical settings. However, the applicability of these estimates to shocks might be limited, e.g., because they do not consider a nonlinear evolution of the filamentation instability and also not a steadily driven instability. In fact, recent numerical simulations of relativistic collisions of the electron-ion plasmas demonstrate the formation of the filamentation-instability-mediated shocks, whose properties do not significantly change with ionto-electron mass ratio between 16 and 1000 (Spitkovsky 2008). Since the characteristics of the magnetic field generation in our simulations are analogous to these observed in relativistic shocks, we similarly expect that the properties of Weibelinstability-mediated nonrelativistic shocks will not be substantially modified for a realistic mass ratio.

Concerning the energy equipartition between electrons and ions, as well as the absence of significant non-thermal tails in the particle spectra, the question arises to what degree residual two-body collisions in the simulation provide thermalization that in the highly collisionless space plasma would not be relevant. In space environments the mean free path for collective plasma interactions is many orders of magnitude smaller than that of two-body collision. In PIC simulations one uses few computational particles to represent very many real electrons or ions and thus introduces artificial collisionality. Would that impact, and possibly prevent, particle pre-acceleration in our simulations?

Coulomb collisions occur with a minimum impact parameter of about one grid-cell length on account of the extended charge distribution used to represent the particle. There are always many ions and electrons within one grid cell, and therefore substantial shielding will occur. Tests indicate that two-body Coulomb interactions are indeed negligible, but random charge imbalances that arise from a low number of particles per cell are possibly not. In fact, they can provide artificial scattering on timescales similar to or shorter than the simulation time for electron velocities $\lesssim 0.03 c$. The post-shock electrons, in particular those in the spectral tail, are always considerably faster than that, and therefore we conclude that in our simulations artificial scattering cannot suppress the formation of non-thermal tails in the electron spectrum.

The authors thank Mark Dieckmann for comments and useful discussions. The work of J.N. is supported by Narodowe Centrum Nauki as research project DEC-2011/01/B/ST9/
03183. M.P. and V.W. acknowledge support through grant PO 1508/1-1 of the Deutsche Forschungsgemeinschaft. The work of A.B. is supported by the project ENE2009-09276 of the Spanish Ministerio de Educacion y Ciencia. Simulations have been performed at the Pleiades facility at the NASA Advanced Supercomputing (NAS) Division.

\section{APPENDIX A \\ SIMULATIONS WITH LOWER ION-ELECTRON MASS RATIO}

The present study is based on simulations that use a reduced ion-electron mass ratio, $m_{i} / m_{e}=50$. To ascertain the effect of this parameter choice on our results, two additional largescale numerical experiments with $m_{i} / m_{e}=20$ have been performed (runs U2 and M2; see Table 1). We find that the main characteristics of the long-time evolution of the systems do not critically depend on the ion-electron mass ratio within the range studied. In both the unmagnetized and magnetized cases, a system of forward and reverse shocks, separated by a $\mathrm{CD}$, is formed. The late-stage structure and nature of the electromagnetic turbulence is analogous to that observed for runs with $m_{i} / m_{e}=50$. Also, the processes of particle-energy dissipation and re-distribution proceed qualitatively in the same fashion. However, the detailed mechanisms of reaching a steady state might be modified or even different if the assumed mass ratio is too low. In the following, we only detail the most important modifications of the system characteristics introduced by a lower-mass ratio.

Figure 16 presents the structure of the collision region at the end of runs $\mathrm{U} 2$ (left) and M2 (right) at time $t=3803 \omega_{\mathrm{pi}}^{-1}$ (to be compared with Figures 3 and 9). Overlaid on the average particle densities and electromagnetic field profiles in Figures 16(a-b) and $(\mathrm{g}-\mathrm{h})$ are the profiles obtained for runs $\mathrm{U} 1$ and M1. The horizontal axis is normalized to the skin depth of dense ions, which is $\lambda_{\mathrm{si}} \approx 36.2$ for $m_{i} / m_{e}=20$. The particle spectra, measured in the CD rest frame, in the downstream region of the forward shock are shown in Figure 17 for run U2. Figure 18 displays spectra in the downstream region of the (a) reverse and (b) forward shock for run M2.

Unmagnetized simulation

1. The initial collision of the tenuous- and dense-plasma slabs leads to magnetic field generation via Weibel-like instabilities, whose level is similar in runs U1 and U2. However, due to their lower mass, the ions are more easily deflected in the magnetic field. Consequently, the ionisotropization rate and the rate of kinetic-energy exchange with electrons are faster. Thus, in run U2 the CD acquires its steady-state velocity earlier, which accounts for a shift of the average profiles for runs U1 and U2 in Figures 16(a) and (b). A substantial part of incoming beam particles is also reflected toward their respective upstream. As a result, the contribution of dense ions to the forwardshock compression is significantly smaller in run U2 (compare solid red and green lines to solid black and blue lines in Figure 16(a)). However, also in this case the tenuous-ion filaments do not fully disintegrate in the shock downstream. Note a slight difference in the electroncompression profile right from the CD (dotted black and blue line in Figure 16(a)) for the two mass ratios.

2. The forward-shock electromagnetic-turbulence structure in run $\mathrm{U} 2$ is analogous to that of run $\mathrm{U} 1$. The role of the 

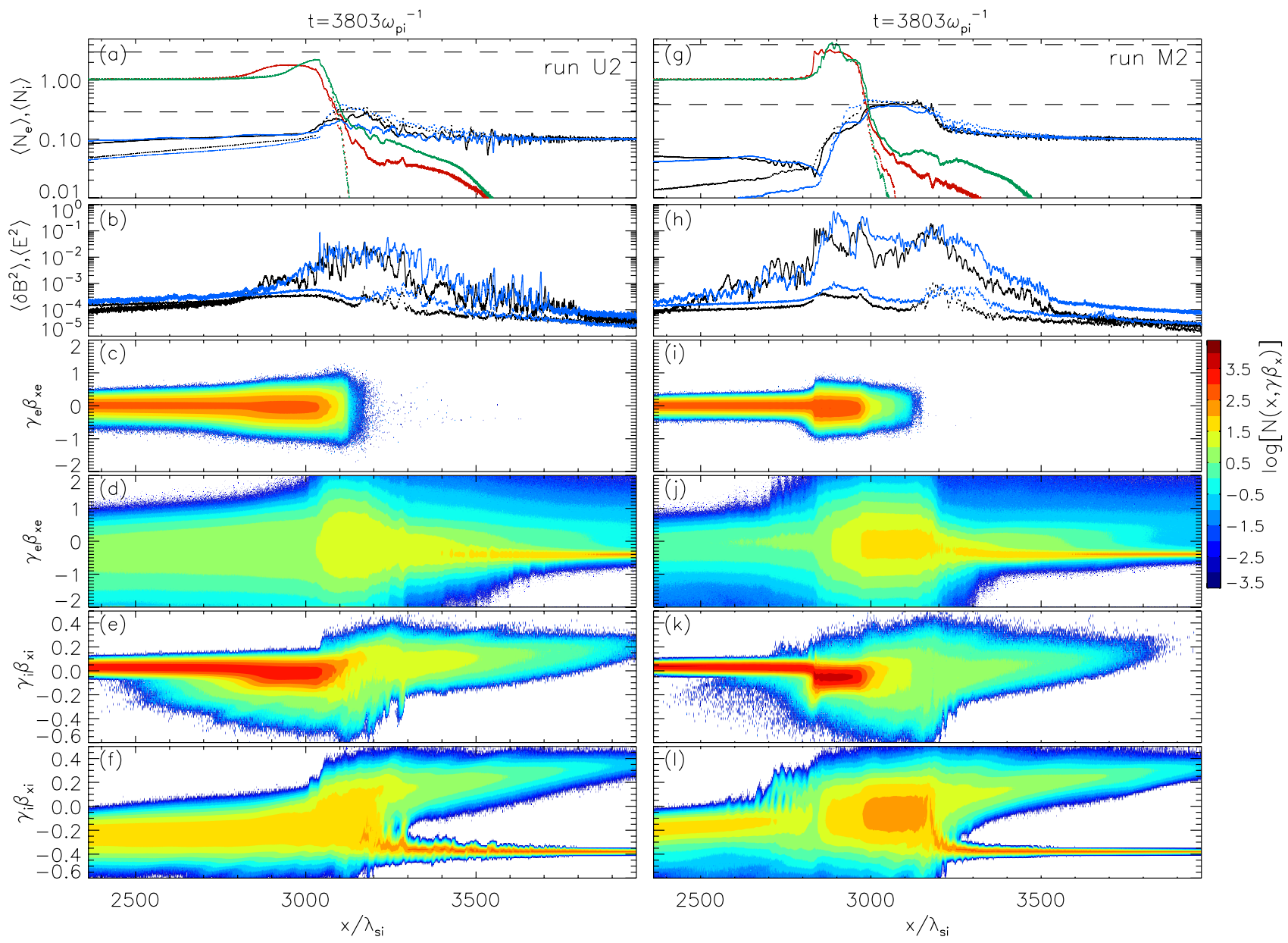

Figure 16. Structure of the collision region at time $t=3803 \omega_{\mathrm{pi}}^{-1}$ for (left) unmagnetized run U2 and (right) magnetized run M2 (see Figure 3). In panels (a) and (g), results for runs $\mathrm{U} 1 / \mathrm{M} 1$ are plotted for $t=3803 \omega_{\mathrm{pi}}^{-1}$ with green lines (dense plasma) and blue lines (tenuous plasma) for comparison. The horizontal dashed lines mark the hydrodynamic compression level for the forward and reverse shocks (see Figures 3 and 8). Panels (b) and (h) overplot the magnetic and electric energy-density profiles for runs $\mathrm{U} 1$ and M1, respectively. The mean magnetic field with energy density of $7.9 \times 10^{-3}$ is subtracted in the magnetic-energy-density profile in panel (h). (A color version of this figure is available in the online journal.)

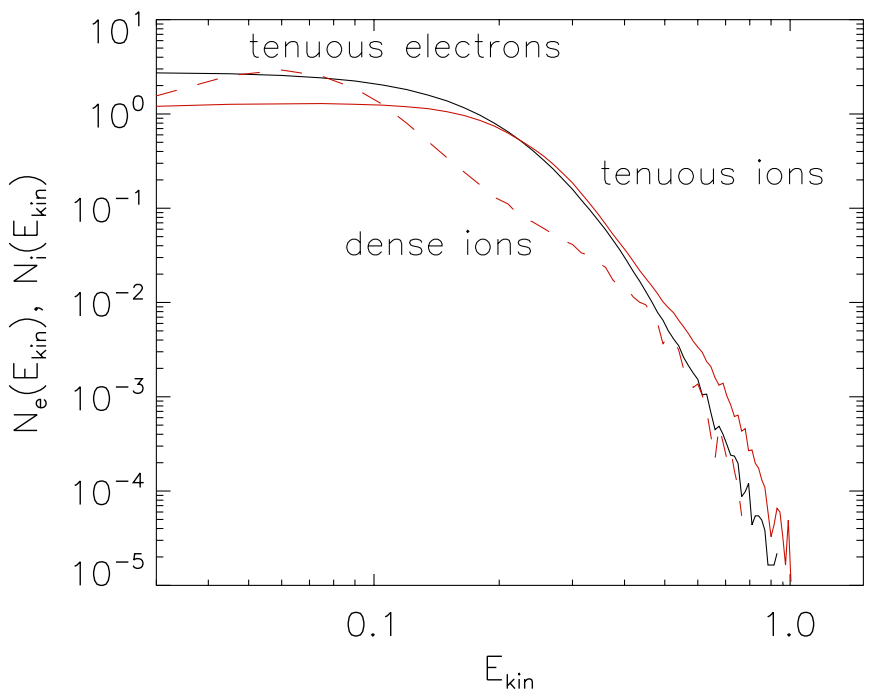

Figure 17. Normalized kinetic-energy spectra of tenuous electrons (black solid line), tenuous ions (red solid line), and the dense ions (red dashed line) downstream of the forward shock at $x / \lambda_{\mathrm{si}} \approx 3100$ and time $t=3803 \omega_{\mathrm{pi}}^{-1}$ for the unmagnetized run $\mathrm{U} 2$, in the $\mathrm{CD}$ rest frame. The ion-electron mass ratio is $m_{i} / m_{e}=20$, and in simulation units $E_{\text {kin }}=0.25(\gamma-1)$ for electrons and $E_{\text {kin }}=5(\gamma-1)$ for ions.

(A color version of this figure is available in the online journal.) dense-ion beam in magnetic-field generation via Weibellike instabilities and in the amplification of the parallel charge-density perturbations is in run U2 shared more equally between the beam of dense ions and the returning tenuous ions (compare Figures 16(e)-(f) and 3(e)-(f)). The magnetic-turbulence level in the filamentation region of the shock precursor $\left(3200 \lesssim x / \lambda_{\text {si }} \lesssim 3500\right)$ is considerably lower for $m_{i} / m_{e}=20$, which results from the higher beam temperatures that cause a serious reduction of the growth rate of filamentation-like modes.

3 . The tenuous-ion beam that permeates the dense-plasma region left from the $\mathrm{CD}$ is much warmer in run $\mathrm{U} 2$ (compare Figures 16(f) and 3(f)). This causes a decrease in the growth rate of the oblique filamentation modes and results in much weaker turbulent precursor to the reverse shock in run U2 (Figure 16(b)). However, an increased efficiency of ion reflection in the $\mathrm{CD}$ region leads to an effective decoupling of the dense-ion beam from the tenuous plasma; the numerous returning dense ions efficiently amplify the magnetic turbulence in the reverse-shock transition. The detailed structure of the reverse shock in run $\mathrm{U} 2$ is thus considerably modified with respect to that observed in run U1 (Figures 16(a) and (b) for $x / \lambda_{\text {si }} \lesssim 3010$ ). However, also for mass ratio $m_{i} / m_{e}=20$, the energy density of the magnetic field left of the $\mathrm{CD}$ is more than an order 

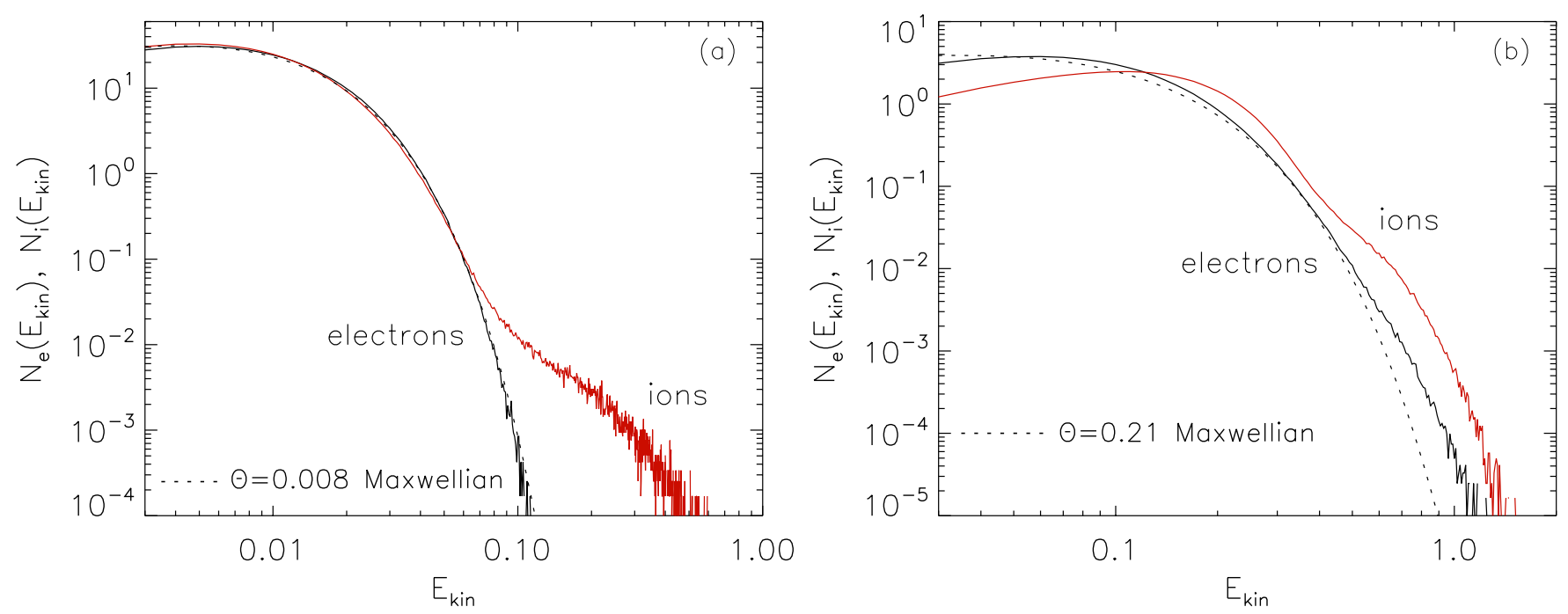

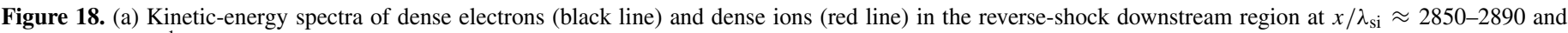

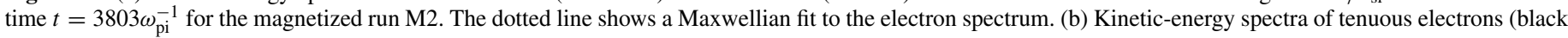

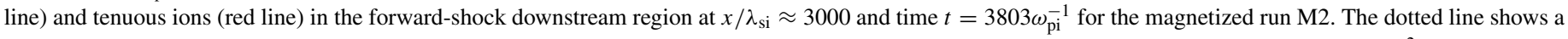
relativistic Maxwellian for comparison. The spectra are shown in CD rest frame and are normalized and expressed in simulation units, in which $m_{e} c^{2}=0.25$.

(A color version of this figure is available in the online journal.)

of magnitude smaller than in the forward-shock region, and the reverse-shock compression does not reach the hydrodynamic limit.

4. Particle spectra downstream of the forward shock (calculated in a slice of width $\sim 20 \lambda_{\text {si }}$, centered at $x / \lambda_{\text {si }} \approx 3100$, i.e., similar location as in run U1) are shown in Figure 17. Whereas the electron and dense-ion spectra display characteristics analogous to those described for run U1, the tenuous-ion distribution resembles that observed downstream of the forward shock in the magnetized simulation, M1. This includes a plateau section in the momentum distribution, which only slightly deviates from isotropy in run U2. The contribution of tenuous ions to the total kinetic energy is, at a level of $38 \%$, the same as in run U1. The dense ions instead carry $17 \%$ only; the difference of $8 \%$ compared to run $\mathrm{U} 1$ is shifted to the electrons, which now account for $44 \%$ of the total kinetic energy. These characteristics suggest that the timescales for particle-energy equilibration might be overestimated in lower-mass ratio simulations.

A slight dependence of the average-density profiles on the ion-electron mass ratio has been reported in Kato \& Takabe (2008). In our simulations, we see a similar effect in the forwardshock profile. Even more significant modifications are observed in the region to the left of the CD. However, the reverse-shock formation has not completed yet by the end of runs U1 and U2.

\section{Magnetized simulation}

1. A reduced mass ratio in run $\mathrm{M} 2$ results in larger Alfvén velocities, which for the tenuous and dense plasma now read $v_{\mathrm{A}, \mathrm{R}}=2.9 \times 10^{-2} c$ and $v_{\mathrm{A}, \mathrm{L}}=8.8 \times 10^{-3} c$, respectively. Thus, the simulation probes a slightly lower AlfvénicMach-number limit (but still $M_{\mathrm{A}} \gg 1$ ), with Mach numbers $M_{\mathrm{Af}} \simeq 13.5$ and $M_{\mathrm{Ar}} \simeq 14.4$ for the forward and reverse shocks, respectively.

2. The characteristic differences introduced by a lower ion mass, and hence larger rate of ion isotropization, which we described above for unmagnetized simulations, are also observed in the magnetized runs M1 and M2. This suggests that it is the ion-beam temperature, and not the slight change in the magnetic field strength the ions experience through an increase of their cyclotron frequency, that is responsible for the observed differences in the development of Weibel-like instabilities in both runs. The level of magnetic turbulence in the forward-shock precursor in run M1 considerably exceeds that in run M2 (Figure 16(h)). This is because in the magnetized case the turbulence is generated exclusively via filamentation-like instabilities, whose growth rates fall off with plasma temperature. Note that the magnetic field decays faster downstream of the forward shock in run $\mathrm{M} 2$.

3. The effects of the warm beams on the system evolution are even more pronounced in the region left of the CD. Instead of the purely transverse filamentation-like mode excited in the dense-plasma region by an energetic tenuousion beam in run M1 (see Section 3.2.1), a much weaker oblique mode is generated in simulation M2. Consequently, the current filaments are weak and their merging does not lead to the strongly nonlinear "magnetic bubble" effects that in run M1 were largely responsible for the efficient decoupling of the colliding ion beams. Nevertheless, also in this case the system can form the reverse-shock transition at an early stage. The shock formation is now mediated by a strong filamentation-like instability due to the large number of dense ions reflected in the collision region.

4. As in run M1, the formation of the reverse-shock transition at late stage in run M2 involves the amplification and compression of a parallel magnetic Bell-like mode. These processes are responsible for the turbulent-field structure at $x / \lambda_{\text {si }} \lesssim 2900$ in Figure $16(\mathrm{~h})$. The parallel left-hand circularly polarized mode has properties similar to these observed in run M1: the wavelength $\lambda \sim 20 \lambda_{\text {si }}$, frequency $\omega \simeq 0.43 \Omega_{i}$, and the phase velocity $v_{\mathrm{ph}} \simeq 1.4 v_{\mathrm{A}, \mathrm{L}}$. Note, however, that the reverse-shock compression in run M2 does not reach the hydrodynamic limit by the end of the simulation. 
5. Particle spectra shown in Figure 18 display characteristics similar to the distributions derived for run M1. However, as is the case of run U2, the energy-equilibration processes proceed with a slightly faster rate for a lower-mass ratio. The kinetic-energy content of the dense electrons downstream of the reverse shock is in run M2 the same within $0.3 \%$ as that of the dense ions, and the electrons are isotropic and thermal (compare a Maxwellian fit to the electron spectrum in Figure 18(a)). Downstream of the forward shock, the tenuous electrons carry $45 \%$ of the total kinetic energy. An analysis of the time evolution of the electron spectra shows an indication of suprathermal tails that were not observed in run M1. Therefore, although the electron spectra in run M2 may be influenced by low statistics at high energies, this result demonstrates that the efficiency of particle injection/pre-acceleration processes might be overestimated in simulations that use a reduced ion-to-electron mass ratio.

\section{APPENDIX B}

\section{DIELECTRIC TENSOR ELEMENTS}

The elements of the dielectric tensor (1) read

$$
\begin{gathered}
a=1-\frac{1+R}{\left(x-Z_{x}\right)^{2}}-\frac{\left(a_{4}+a_{5}+a_{3} R\right) \beta_{1}^{2}}{\left(x \beta_{1}+Z_{x} \beta_{2}\right)^{2}}, \\
b=1-\frac{Z_{x}^{2}}{x^{2} \beta_{1}^{2}} \\
-\frac{R}{x^{2}}\left[\frac{\left(x-Z_{x}\right)^{2} / R}{\left(x-Z_{x}\right)^{2}-\Omega_{B}^{2}}+\frac{\left(x-Z_{x}\right)^{2}}{\left(x-Z_{x}\right)^{2}-R^{2} \Omega_{B}^{2}}-B\right], \\
c=\frac{i R^{2} \Omega_{B}}{x^{2}}\left[\frac{\left(x-Z_{x}\right) / R^{2}}{\left(x-Z_{x}\right)^{2}-\Omega_{B}^{2}}-\frac{x-Z_{x}}{\left(x-Z_{x}\right)^{2}-R^{2} \Omega_{B}^{2}}+C\right],
\end{gathered}
$$

with

$$
\begin{aligned}
& B= \\
& \frac{\left(a_{4}+a_{5}+a_{3} R\right)\left(x \beta_{1}+Z_{x} \beta_{2}\right)^{4}-R\left(a_{3}+\left(a_{4}+a_{5}\right) R\right) \beta_{1}^{2}\left(x \beta_{1}+Z_{x} \beta_{2}\right)^{2} \Omega_{B}^{2}}{R\left(1+R^{2}\right) \beta_{1}^{2}\left(x \beta_{1}+Z_{x} \beta_{2}\right)^{2} \Omega_{B}^{2}-R^{3} \beta_{1}^{4} \Omega_{B}^{4}-R\left(x \beta_{1}+Z_{x} \beta_{2}\right)^{4}}
\end{aligned}
$$

$$
\begin{aligned}
C= & \frac{\left(a_{4}+a_{5}\right) \beta_{1}\left(x \beta_{1}+Z_{x} \beta_{2}\right) / R^{2}}{\left(x \beta_{1}+Z_{x} \beta_{2}\right)^{2}-\beta_{1}^{2} \Omega_{B}^{2}} \\
& -\frac{a_{3} \beta_{1}\left(x \beta_{1}+Z_{x} \beta_{2}\right)}{\left(x \beta_{1}+Z_{x} \beta_{2}\right)^{2}-R^{2} \beta_{1}^{2} \Omega_{B}^{2}} .
\end{aligned}
$$

In these expressions, $Z_{x}=k_{x} v_{L} / \omega_{\mathrm{pe}, L}$ is the reduced flowaligned wavevector, $R$ is the electron-to-ion mass ratio, and $\Omega_{B}=\Omega_{e} / \omega_{\mathrm{pe}, L}$ is the reduced electron cyclotron frequency. The parallel magnetic mode appears in the reverse-shock precursor in the late stage of our magnetized simulations M1 and M2 (Figure 12). However, the mode can also be observed in the linear spectrum of the early-stage turbulence (Figure 6). We provide parameters $a_{n}$ and $\beta_{n}$ for both setups in Table 2.
Table 2

Parameters $a_{n}$ and $\beta_{n}$ of the Dielectric Tensor for the Setups of Figures 6 and 12; Run M1

\begin{tabular}{llllll}
\hline \hline Figures & $a_{3}$ & $a_{4}$ & $a_{5}$ & \multicolumn{1}{c}{$\beta_{1}$} & \multicolumn{1}{c}{$\beta_{2}$} \\
\hline 6 & 0.1 & 0.01 & 0.09 & 0.0354 & 0.354 \\
12 & 0.05 & 0.005 & 0.045 & 0.0354 & 0.3 \\
\hline
\end{tabular}

\section{REFERENCES}

Amano, T., \& Hoshino, M. 2009, ApJ, 690, 244

Amano, T., \& Hoshino, M. 2010, Phys. Rev. Lett., 104, 181102

Bell, A. R. 2004, MNRAS, 353, 550

Blandford, R., \& Eichler, D. 1987, Phys. Rep., 154, 1

Bret, A. 2007, Comput. Phys. Commun., 176, 362

Bret, A. 2009, ApJ, 699, 990

Bret, A., Dieckmann, M. E., \& Deutsch, C. 2006, Phys. Plasmas, 13, 082109

Bret, A., Firpo, M.-C., \& Deutsch, C. 2005, Phys. Rev. Lett., 94, 115002

Bret, A., Gremillet, L., \& Benisti, D. 2010, Phys. Rev. E, 81, 036402

Buneman, O. 1993, in Computer Space Plasma Physics: Simulation Techniques and Software, ed. H. Matsumoto \& Y. Omura (Tokyo: Terra), 67

Burgess, D., \& Scholer, M. 2007, Phys. Plasmas, 14, 012108

Cargill, P. J., \& Papadopoulos, K. 1988, ApJ, 329, L29

Chang, P., Spitkovsky, A., \& Arons, J. 2008, ApJ, 674, 378

Classen, H.-T., \& Mann, G. 1997, A\&A, 322, 696

Dieckmann, M. E., Kourakis, I., Borghesi, M., \& Rowlands, G. 2009, Phys. Plasmas, 16, 074502

Dieckmann, M. E., Murphy, G. C., Meli, A., \& Drury, L. O. C. 2010, A\&A, 509, A89

Fiore, M., Silva, L. O., Ren, C., Tzoufras, M. A., \& Mori, W. B. 2006, MNRAS, 372, 1851

Fonseca, R. A., Martins, S. F., Silva, L. O., et al. 2008, Plasma Phys. Control. Fusion, 50, 124034

Forslund, D. W., \& Freidberg, J. P. 1971, Phys. Rev. Lett., 27, 1189

Forslund, D. W., \& Shonk, C. R. 1970, Phys. Rev. Lett., 25, 1699

Frederiksen, J. T., Hededal, C. B., Haugboelle, T., \& Nordlund, A. 2004, ApJ, $608, \mathrm{~L} 13$

Gargaté, L., \& Spitkovsky, A. 2012, ApJ, 744, 67

Gary, S. P. 1984, J. Geophys. Res., 89, 179

Goldman, M. V. 1984, Rev. Mod. Phys., 56, 709

Greenwood, A. D., Cartwright, K. L., Luginslund, J. W., \& Baca, E. A. 2004, J. Comput. Phys., 201, 665

Hededal, C. B., Haugboelle, T., Frederiksen, J. T., \& Nordlund, A. 2004, ApJ, 617, L107

Kato, T. N., \& Takabe, H. 2008, ApJ, 681, L93

Kato, T. N., \& Takabe, H. 2010, ApJ, 721, 828

Kazimura, Y., Califano, F., Sakai, J.-I., et al. 1998a, J. Phys. Soc. Japan, 67, 1079

Kazimura, Y., Sakai, J. I., Neubert, T., \& Bulanov, S. V. 1998b, ApJ, 498, L183

Krauss-Varban, D., \& Omidi, N. 1991, J. Geophys. Res., 96, 17715

Lembège, B., Giacalone, J., Scholer, M., et al. 2004, Space Sci. Rev., 110, 161

Lembège, B., Savoini, P., Hellinger, P., \& Trávníček, P. M. 2009, J. Geophys. Res., 114, 3217

Matsumoto, Y., Amano, T., \& Hoshino, M. 2012, ApJ, 755, 109

McClements, K. G., \& Fletcher, L. 2009, ApJ, 693, 1494

Medvedev, M. V. 2006, ApJ, 651, L9

Medvedev, M. V., Fiore, M., Fonseca, R. A., Silva, L. O., \& Mori, W. B. 2005, ApJ, 618, L75

Medvedev, M. V., \& Loeb, A. 1999, ApJ, 526, 697

Murphy, G. C., Dieckmann, M. E., Bret, A., \& Drury, L. O’C. 2010, A\&A, 524,84

Niemiec, J., Pohl, M., Bret, A., \& Stroman, T. 2010, ApJ, 709, 1148

Niemiec, J., Pohl, M., Stroman, T., \& Nishikawa, K.-I. 2008, ApJ, 684, 1174

Nishikawa, K.-I., Hardee, P. E., Hededal, C. B., \& Fishman, G. J. 2003, ApJ, 642, 1267

Nishikawa, K.-I., Hardee, P. E., Richardson, G., et al. 2003, ApJ, 595, 555

Nishikawa, K.-I., Niemiec, J., Hardee, P. E., et al. 2009, ApJ, 698, L10

Papadopoulos, K. 1988, Ap\&SS, 144, 535

Polomarov, O., Kaganovich, I., \& Shvets, G. 2008, Phys. Rev. Lett., 101, 175001

Quest, K. B. 1988, J. Geophys. Res., 93, 9649

Ren, C., Blackman, E. G., \& Fong, W. 2007, Phys. Plasmas, 17, 012901

Reville, B., Kirk, J. G., \& Duffy, P. 2006, Plasma Phys. Control. Fusion, 48,1741

Riquelme, M. A., \& Spitkovsky, A. 2009, ApJ, 694, 626 
Riquelme, M. A., \& Spitkovsky, A. 2011, ApJ, 733, 63

Shvets, G., Polomarov, O., Khudik, V., Siemon, K., \& Kaganovich, I. 2009, Phys. Plasmas, 16, 056303

Silva, L. O., Fonseca, R. A., Tonge, J. W., et al. 2003, ApJ, 596, L121

Spitkovsky, A. 2008, ApJ, 673, L39

Stasiewicz, K., \& Ekeberg, J. 2008, ApJ, 680, L153

Stroman, T., Pohl, M., \& Niemiec, J. 2009, ApJ, 706, 38

Stroman, T., Pohl, M., Niemiec, J., \& Bret, A. 2012, ApJ, 746, 24
Sugiyama, T. 2011, Phys. Plasmas, 18, 022302

Tzoufras, M., Ren, C., Tsung, F. S., et al. 2006, Phys. Rev. Lett., 96, 105002

Umeda, T., Yamao, M., \& Yamazaki, R. 2009, ApJ, 695, 574

Weibel, E. S. 1959, Phys. Rev. Lett., 2, 83

Wiersma, J., \& Achterberg, A. 2004, A\&A, 428, 365

Winske, D., \& Leroy, M. M. 1984, J. Geophys. Res., 89, 2673

Winske, D., Thomas, V. A., Omidi, N., \& Quest, K. B. 1990, J. Geophys. Res., 95,18821 